\title{
Les trois amours platoniciens ou la philosophie à hauteur d'homme
}

Anca Vasiliu

\section{(2) OpenEdition}

1 Journals

Édition électronique

URL : https://journals.openedition.org/philosant/943

DOI : 10.4000/philosant.943

ISSN : 2648-2789

Éditeur

Éditions Vrin

\section{Édition imprimée}

Date de publication : 1 novembre 2012

Pagination : 237-269

ISBN : 978-2-7574-0400-3

ISSN : 1634-4561

\section{Référence électronique}

Anca Vasiliu, «Les trois amours platoniciens ou la philosophie à hauteur d'homme », Philosophie antique [En ligne], 12 | 2012, mis en ligne le 01 novembre 2018, consulté le 02 décembre 2022. URL: http://journals.openedition.org/philosant/943 ; DOI : https://doi.org/10.4000/philosant.943

\section{(c) $(7)(9$}

Creative Commons - Attribution - Pas d'Utilisation Commerciale - Pas de Modification 4.0 International - CC BY-NC-ND 4.0

https://creativecommons.org/licenses/by-nc-nd/4.0/ 


\section{LES TROIS AMOURS PLATONICIENS OU LA PHILOSOPHIE À HAUTEUR D'HOMME}

Anca VASILIU

Centre de recherches sur la pensée antique, CNRS, Paris

RÉSUMÉ. Consacrée à l'analyse de quelques passages du Phèdre, du Banquet et de l'Alcibiade, cette étude rappelle la définition platonicienne du rôle pédagogique de l'amour dans la théorie de la connaissance et dans la pratique du langage. Sa thèse est que le modèle anthropologique et le modèle épistémologique ne peuvent pas être séparés dans la philosophie platonicienne. « Parler » constitue à la fois une communication et une ostension, donc la transitivité du langage n'est pas exempte d'une nécessaire vérité de l'acte, même si cette vérité est celle du phénomène, non celle de l'essence, de la forme ou du principe. Les conséquences de cette unité (présentée comme une rencontre entre la rhétorique et la dialectique) sont d'ordre éthique autant que d'ordre métaphysique. D'une part, l'autonomisation du langage pose comme préalable un rapport de vérité à l'égard de l'autre dans toute détermination d'identité du sujet. Ce rapport de vérité s'exprime ici sous la forme initiatique d'une «mise en présence » : l'accès à soi est conditionné par la transcendance du tout-autre. Acquise par la présence amoureuse à l'égard de l'autre, l'identité n'est donc pas close sur elle-même mais se définit comme relation. D'autre part, la maîtrise d'un langage adéquat au phénomène érotique suppose le dépassement de l'interprétation (« péché contre la mythologie ») et une sortie de l'opposition des contraires (corps-âme) par la limite assumée (ici la « faiblesse des mots »), sans appeler nécessairement à l'harmonie universelle qui, traditionnellement, cache la domination, voire la suppression du sujet.

SUMMARY. This paper focuses on some passages from Plato's Phaedrus, Symposium and Alcibiades, examining at the role played by love in the pursuit of knowledge and in the art of rhetoric. I argue that the anthropological insights cannot be separated from the epistemological line. Speech is both an act of communication and a demonstration, so that the transitive character of language is not deprived of a necessary performance of truth. The implications of this unit (analyzed as the encounter between rhetoric and dialectic) are both ethical and metaphysical. On the one hand, it presupposes a truthful relationship to the other, starting from a clear identification of the speaking subject. This relationship of truth takes the form of an initiation. On the other hand, the mastery of an appropriate language goes beyond interpretation ("sin 
against mythology") and proposes an alternative to the exclusion of opposites through an appropriation of the limits of language (the "weakness of words"). But this attitude does not necessarily fall back on the idea of transcendent harmony, which traditionally hides the actual suppression of the subject. 
Un fait a deux faces parce qu'un fait n'est pas seulement un événement dans le monde, mais l'affirmation d'un événement, la mise en mots du monde (the wording of the world). Stanley Cavell, Sens de Walden *

Dans un texte rebattu du Phèdre (264c) Platon affirme la nécessité pour tout discours, autrement dit pour toute ouvre littéraire de la pensée,

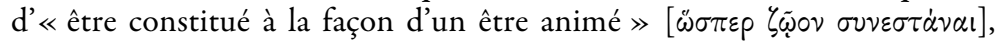
d'avoir un corps qui ait une partie centrale, une tête, des membres, bref des éléments qui soient solidaires les uns des autres et du tout, se convenant

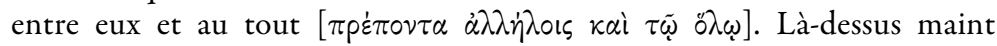
critique s'étonne que Platon ait si mal appliqué un précepte si bien formulé : comment se fait-il que, dans une première partie, le Phèdre traite de l'amour et de la beauté, puis dans une seconde, de la rhétorique opposée à la dialectique ? Certains en prennent bravement leur parti : Platon était vieux quand il écrivit le Phèdre, et son art avait perdu de sa souplesse. La plupart font des efforts désespérés pour découvrir une cohésion à laquelle ils ne croient guère; ils cherchent surtout à subordonner à l'autre une des deux parties, espérant ainsi trouver dans la partie dominante le principe de l'unité de l'ensemble.

Si j’ai cité ce texte de Léon Robin, extrait de sa longue étude qui introduit l'édition-traduction du Phèdre aux éditions Les Belles Lettres en $1933^{1}$, c'est parce qu'il résume parfaitement, me semble-t-il, la situation «classique » dans laquelle se trouve le discours philosophique ancien. Mettant en lumière ce que les historiens ont considéré comme la faille du Phèdre, ce passage montre implicitement que le langage de la philosophie hésite encore chez Platon entre un modèle anthropologique et un modèle épistémologique, entre une pratique singulière de la parole comme une expression propre à chaque individu, et un exercice spécifique d'école, pour lequel le langage de la philosophie est le moyen approprié à la transmission d'un savoir universel et d'une science applicable à tout objet donné. Encore autrement dit, il y aurait là l'expression d'une hésitation fondatrice entre

* The Senses of Walden, 1972, trad. française par B. Rival et O. Berrada, Théâtre Typographique, Paris, 2007, p. 52. Sur Walden de H. D. Thoreau.

1. Robin 1933, p. XXVI (= Vicaire 1985, p. XXXVI). 
un acte de parole qui pratique sciemment la possession d'un pouvoir et un exercice réflexif de la parole qui joue des feux de l'intelligence dans l'art de dire et de faire voir sans viser nécessairement une retombée immédiate extérieure à cet acte de parole, et donc sans instrumentaliser d'emblée le langage. Mais, en formulant cette critique à l'adresse d'une composition jugée hétéroclite (une critique inaugurale de la manipulation ?), l'exégète platonicien de la première moitié $\mathrm{du} \mathrm{XX}^{\mathrm{e}}$ siècle laisse entendre aussi quelle serait selon lui la vocation à laquelle le philosophe antique voue l'être humain, du moins ce qu'il considère comme le meilleur de l'être humain, et s'inscrit ainsi lui-même, de ce fait, dans le temps long d'une certaine postérité de l'Académie. En effet, ce qui permet à un homme de manifester son humanité propre et singulière serait, selon l'exégète de Platon, le pouvoir acquis de parler selon les règles de l'art qui convient au sujet abordé. L'homme accomplirait sa nature d'homme lorsqu'il adresse à autrui un discours non seulement cohérent et raisonnable, mais encore plus semblable en tout point à lui-même que ne le serait son portrait peint ou sculpté, puisque le discours est « vrai » et « vivant », ou « vrai » puisque « pareil à un vivant ». Aussi la parole proférée est-elle image parfaite de celui qui parle, image de ce qu'il y a en lui de plus vivant : son âme et son intelligence. Le discours est ainsi à l'orateur antique ce qu'un fils doit être pour son père : une image à la fois semblable et vivante, à la fois preuve de sa vie et expression accomplie de sa pensée.

La question « comment parler au sujet de... l'amour, la vertu, le Beau etc. ? », comment transmettre le contenu d'une définition et, en somme, comment transmettre une science, ne saurait par conséquent être séparée dans ce contexte de la nécessité de savoir faire acte de parole en dehors de toute transmission d'une définition, de savoir répondre et discourir en chaque circonstance donnée, et d'en faire acte comme d'une parole propre autonome, même si celle-ci est adressée et circonscrite à une situation. Car prendre la parole ne signifie pas seulement communiquer un savoir, mais consiste aussi, et surtout pour les Anciens, à pouvoir se montrer en tant qu'homme qui parle à autrui en prouvant ainsi la capacité de dire au plus juste sa pensée et en assumant les effets de la puissance qui s'exerce immanquablement lorsqu'on prend la parole devant autrui. Encore faut-il se rappeler qu'un homme qui prend la parole est pour les contemporains de Platon un homme libre, non un esclave ; et partant se demander si la parole, vraie ou fausse, lui appartient puisqu'il a appris l'art du langage, ou si tout homme qui parle, qu'il soit « simple » ou homme de métier, ne serait pas, toujours, une caisse de résonance, une voix d'emprunt pour la parole d'un autre - ancêtre, Muse ou dieu -, dont il répète délibérément ou à son insu les propos, voire le raisonnement et les figures conventionnelles de l'expression. L'identité de celui qui parle révèle-t-elle alors l'identité vivante d'un 
être singulier - Socrate, Phèdre, Alcibiade ou Théétète -, ou se fait-elle l'écho d'un raisonnement à portée universelle qui emprunte une voix particulière pour pouvoir s'exprimer et se faire entendre dans une situation donnée?

C'est à partir de cette perspective que je voudrais exposer, sans ignorer l'arrière-goût politique qui demeure sous le terme anachronique d'humanisme, terme que ne saurait traduire l'idéal d'une «philosophie à hauteur d'homme », la vocation à laquelle Platon semble vouer l'être humain. Du moins, ce qu'un certain humanisme classique considère comme la définition platonicienne du «meilleur » dans l'être humain. En effet, Platon met en ouvre un moyen par lequel il (nous) devient possible de lire dans ses dialogues une réponse à cette question encombrante pour la pensée : quid d'être-un-homme, ou du moins, à défaut, comment, quomodo, être « homme » à partir de l'identité propre de chaque individu humain ? Non ce qu'est un homme, car la réponse réclamerait alors de définir l'âme, selon l'assertion bien connue de Socrate dans Alcibiade ${ }^{2}$, mais comment manifester l'acte d'être un « homme » et non seulement d'être un «vivant ». Sans déroger au principe de la connaissance des formes qui interdit tout accès au singulier et à l'identité individuelle en raison de leur soumission au multiple et aux lois du devenir, et sans y consacrer par conséquent un exposé voué explicitement à la détermination de l'être individuel, unique et singulier, le moyen utilisé par Platon consiste en une analyse serrée de l'acte conjoint de penser et de parler. Cette analyse, récurrente dans ses textes, est plus particulièrement présente dans les dialogues dits « socratiques », où le recours à l'altérité constitue le principe régulateur de la connaissance de soi - selon la description du rapport catoptrique à soi par l'autre, description dont l'Alcibiade est encore la référence classique ${ }^{3}$. Le philosophe cherche ainsi en toute chose, et non au sujet de l'homme en premier, ce qui permet à un homme de penser et d'exprimer l'identité de soi et, sur le même principe, l'identité de chaque objet singulier en ce que celui-ci a de commun précisément avec la possibilité d'être exposé et d'être connu tant par le voũs que dans le $\lambda o ́ \gamma \circ \varsigma$, tant dans son intelligibilité séparée du contingent que par une « descente » dans le raisonnement et dans la langue. Les philosophes « platoniciens » d'un humanisme prolongé jusque dans la modernité ont voulu voir dans cette analyse conjointe de la pensée et du langage la raison philosophique qui justifiait l'affirmation de l'homme comme « mesure de toute chose »: «mesure » de ce qui est et de ce qui n'est pas, de ce qui porte sens durablement et de ce qui n'est qu'un hochet transitif dans la

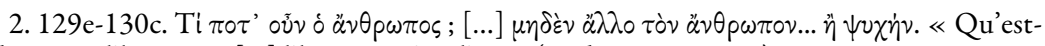
ce donc que l'homme ? [...] l'homme, c'est l'âme. (trad. Croiset 1920).

3. Platon, Alc. 132c sq. 
vacuité du phénomène sensible de la langue. La sublimité qu'ils y ont attachée ne pouvait que générer la réaction d'une autre forme de sublime, l'excès de la négation, à partir d'une version nietzschéenne de l'humanisme dont les racines sont tout aussi «platoniciennes » que celles du classicisme de la Renaissance et des Lumières.

Mais je ne m'engagerai pas ici dans un dialogue avec les uns ou les autres des représentants du platonisme moderne. Je voudrais simplement situer l'analyse conjointe des actes de penser et de parler dans le contexte littéral de quelques dialogues socratiques et montrer cette conjonction à l'œuvre à partir d'un des sujets abordés : celui de la définition de l'amour. Sujet privilégié, certes, puisque l'amour « socratique » n'est pas un «objet » dont on recherche la possession en le définissant, mais un moyen spécifique pour assumer à l'œuvre ce double apprentissage - de l'art de connaître et de l'art de parler. Situer cette analyse ne va toutefois pas sans une mise en perspective propre. Celle que je proposerai ici provient des questions actuelles concernant la définition du statut du langage : relativisme d'une vérité de la phrase? ou affirmation d'une vérité de sens qui implique une double présence et qui suppose l'horizon d'une éthique ? Il me semble pertinent d'analyser les dialogues sous cet angle puisque Platon lui-même formule à sa manière cette alternative à l'égard du langage. La faille du Phèdre s'avère être dans ce cas la conséquence d'une construction bipartite volontaire, pour montrer le caractère artificiel d'une séparation des genres dans le périmètre du sujet choisi pour cette fin.

Prenons donc comme point de départ le dialogue cité par Léon Robin. La leçon de Socrate à Phèdre dresse une ligne de partage nette entre d'une part le « secret » de l'art de parler (ce que Socrate appelle avec ironie l'in-

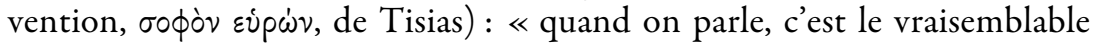

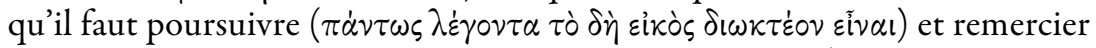

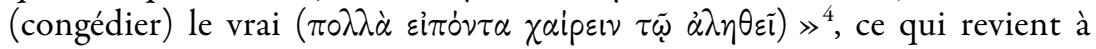

4. Phaedr. 272e (je traduis) et $273 \mathrm{~b}$ pour la définition de l'art de Tisias. La critique de la vraisemblance apparaît indirectement dans le Sophiste dans la définition de l'image comme l'instrument-maitre du sophiste (240a sq.), alors qu'elle est formulée amplement et directement dans le Phèdre (272b-273b), dans le passage que je viens de citer. Le contexte est celui de la définition de l'art oratoire ( $\left.\lambda \varepsilon \gamma o \mu \varepsilon v \eta \varsigma \lambda \partial^{\prime} \gamma \omega \nu \tau \dot{\varepsilon} \chi \nu \eta \varsigma\right)$ tel qu'il serait pratiqué, selon Socrate, dans les tribunaux et dans l'agora. On peut y voir le signe d'une tradition sophistique dont on retrouve la trace aussi chez Aristote (Metaph. 1015a5 et Rhet. II, 1402a13-14), chez Démétrios (Eloc. 120) et chez Antiphon (Tetral. I, $\beta, 8$ ). L'usage du vraisemblable est théorisé et défendu dans un chapitre qui lui est consacré exclusivement dans la Rhétorique à Alexandre (7, 4-14). Dans ce dernier traité, l'eikos tient une place importante parmi les «Moyens de persuasion issus des paroles, des actions et des hommes » et est mis en connexion avec l'usage des exemples (paradeigmata). Voir pour ce dernier Chiron 2002 (p. LXX- 
dire, en d'autres termes, qu'il est juste de plaider même la cause du loup ${ }^{5}$, et

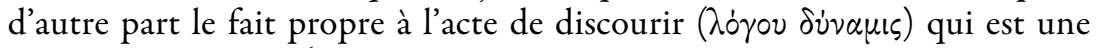
forme de psychagogie $e^{6}$ et qui, à ce titre, signifie connaître l'âme et la montrer en paroles selon la condition qui fait qu'un homme soit homme accompli, c'est-à-dire un maître absolu de ses passions. L'acte de parler exerce ainsi un double pouvoir qui se meut entre la condition irrépressible de l'homme en tant que «lieu où l'être se dit », pour parler en des termes heideggeriens connus (termes qui rappellent d'ailleurs la condition de l'affirmation ontologique chez Parménide), et qui rend le vrai impossible à cacher sur le visage ou dans l'expérience immédiate du sensible, et une action propre aux « mouvements de la langue », pour emprunter une expression à Austin ${ }^{7}$, action au cours de laquelle tout, vrai ou faux, peut être dit à condition de séparer d'emblée dire et montrer.

Sans répéter à l'égard d'un autre objet herméneutique les propos de Barbara Cassin consacrés à ce que signifie en fin de compte la décision du sens dans l'acte de parler (legein), selon les affirmations du livre Gamma de la Métaphysique d'Aristote ${ }^{8}$, décision qui légitime le fait d'être un homme, je voudrais déterminer l'acte de parole comme étant chez Platon l'opérateur de ce double pouvoir du langage, dire et montrer, sous la modalité d'un double dévoilement : dévoilement de l'identité singulière de l'homme qui parle en son nom (Socrate enlève alors le voile qui couvrait sa tête lors de son premier discours en réponse à Phèdre, alias Lysias); et dévoilement aussi de l'identité sémantique de l'objet de référence qui apparaît comme (une) présence signifiante dans chaque affirmation de son existence et de son sens - que cette affirmation soit celle d'une nomination abstractive et universaliste (l'être ou le Bien), ou qu'elle intervienne au cours d'une narration qui décrit et signifie le passage à l'acte, opère avec l'individuel (comme avec des monades enfermées dans le ton épique) et vise le réel (ou la production du réel), même si nous savons que la narration consiste chez Platon le plus souvent en un mythos. Parler en son nom, même lorsqu'il s'agit de rapporter les paroles d'un autre que soi-même, et mettre l'objet en présence par la parole qui s'y réfère comme gage d'accès à son identité sémantique, sont donc les deux faces d'un fait unique accompli par le discours.

Ce fait, appelé de nos jours «vérité de langue » ou affirmation valide lors d'un acte de langage (Socrate s'adresse directement à Phèdre et vise

LXXV de l'Introduction pour les rapprochements entre la tradition sophistique et la théorie du vraisemblable).

5. Proverbe cité par Socrate, Phaedr. 272 c.

6. Ibid. $271 \mathrm{c}$.

7. « Plaidoyer pour les excuses », dans Austin 1994, p. 139.

8. « Parle, si tu es un homme », dans Cassin \& Narcy 1989, p. 9-60. 
Phèdre en personne en définissant l'amour), ne peut pas être séparé de l'expérience métalinguistique (ici : le fait de se montrer), et cette expérience impose une confrontation et demande en même temps une tentative d'ajustement ou d'adéquation entre langage et évidence - évidence du visible en premier lieu, puisque la vision est l'expérience la plus courante. Le dédoublement entre auteur et acteur du texte, qui «fait recette » chez Platon, exprime la nécessité de prendre en compte cette expérience, d'en prendre acte dans le courant de l'affirmation et de la définition de chaque identité, même (et surtout) lorsqu'il s'agit de structurer un concept. Or le dédoublement (auteur-acteur), qui situe en outre le processus d'ajustement ou d'adéquation dans une tension, traduit dans le contexte platonicien la nécessité d'une « initiation ». L'apprentissage d'un savoir est aussi une initiation au «mystère » de la langue, i.e. à l'art du discours. D’où la nécessité d'un dédoublement quasi permanent qui se produit aussi à l'égard de la personne même de l'acteur mis en scène par l'auteur, acteur qui rapporte alors les paroles d'un tiers: Phèdre lit dans le dialogue éponyme le discours de Lysias (comme une «initiation » pour lui à la parfaite rhétorique) ; Socrate rapporte dans le Banquet les paroles de Diotime (comme une « initiation » à l'éloge de l'amour parfait) etc. Si cette structure du texte platonicien tient d'un genre littéraire particulier qui ne nous intéresse pas dans cette étude, la mise en situation de la parole engendrée par cette structure propre à la médiation nécessaire dans une initiation me semble en revanche relever du statut que Platon veut donner à l'acte de la parole dans certaines circonstances et à l'égard de certains objets particuliers du discours. Le danger encouru est celui de déplacer l'intérêt depuis le terrain de l'identité philosophique vers le terrain du jeu littéraire, un jeu de places substituées et de places prises dans l'esprit du lecteur. Mais si on prête l'attention à ce glissement facile et si on redresse la barre en tenant le cap de l'enjeu philosophique, et nécessairement éthique, du dialogue platonicien, ce jeu de rôles et de situations de parole peut devenir l'instrument d'une analyse dont l'objet est justement la constitution du langage philosophique à partir du langage ordinaire des hommes, le sens premier des mots avec lequel Socrate aiguillonne les citoyens d'Athènes et talonne ses disciples pour leur apprendre à raisonner.

Je prends comme «thèse » la définition de ces deux faces du fait accompli par un discours: (a) que le langage philosophique ne constitue pour Platon rien d'autre que l'instrument dont l'appropriation et l'usage permettent à un homme d'être pleinement lui-même, en intégrant et en assumant le contenu qu'il assigne à chaque mot comme une expression, et une ostension en même temps, de sa pensée et (b) que le langage philosophique se définit comme une maîtrise de la pratique de l'acte de parole en situation, afin de mettre l'individu qui parle en rapport avec lui-même (en situation 
de dominer ses passions, en l'occurrence) dès lors qu'il se dévoile comme objet phénoménal en toute circonstance qui le concerne directement ${ }^{9}$. Une analyse serrée de l'acte conjoint de penser et de parler - j'entends une analyse de l'acte en même temps que de la situation engendrée par l'acte - permet ainsi de baliser le terrain en déplaçant l'intérêt « classique » pour la théorie de la connaissance par les Formes vers une visée plus étroite, immanente à l'acte d'exister : à savoir, le rapport de détermination et d'implication mutuelle du phénomène et du langage.

Sous couvert d'un Socrate curieux de tout en même temps qu'entiché de discours, Platon cherche à l'égard de toute chose, et non au sujet de l'homme en premier, ce qui permet à un homme de penser et d'exprimer l'identité de chaque objet singulier en ce que celui-ci a de commun précisément avec la possibilité d'être exposé, « réduit » à l'expression. Ainsi, tout objet (qu'il soit être ou action dans le choix entre immanent et transcendant) peut être connu non seulement comme une altérité par rapport à celui qui le saisit, mais aussi comme une identité à part entière grâce à la faculté appropriée $\mathrm{du}$ nous, par une intellection qui fait de lui l'objet de la communication d'un contenu assigné par l'intellect à travers le logos. L'identité est ainsi accessible aussi longtemps que subsiste la possibilité de constituer un objet du logos ou, si l'on préfère, autant que demeure dans le legein, dans l'acte de prédication, un objet de la dénomination et de l'énonciation à partir de chaque objet singulier de l'expérience. Mais la méthode dialectique pratiquée par Socrate ne produit son véritable effet bénéfique sur la pensée - un effet d'éclaircissement du sens par l'art des distinctions entre objets et « vérités » à l'égard des objets - que dans le cadre d'une ontologie dont l'être premier n'est autre que l'homme lui-même et non quelque objet que ce soit dont on cherche la détermination dans l'acte d'exister et on arrête la connaissance sur les principes de production. Si l'objet de l'expérience devient un objet sur lequel on prend pouvoir par l'expression, cette appropriation de l'objet ne sert en réalité qu'à fixer les limites de l'identité de l'objet par rapport à celui qui parle et qui connaît ou prétend connaitre cette identité. L'identité est certes conçue en rapport à l'altérité, mais à travers une objectivation par le logos, par une pratique appropriée de l'acte du langage.

9. Il va de soi que la possibilité du mensonge ou du faux témoignage ne trouve pas de place dans ce contexte, ou alors, si mensonge il y a, il est de l'ordre d'une parole rapportée ou d'une « démonstration d'école » sans fondement ontologique, tel le discours de Lysias rapporté par Phèdre au début du dialogue éponyme. Précisons, pas ailleurs, que cette position ne nous semble pas contradictoire avec la démonstration anti-parménidienne du Sophiste concernant la possibilité de parler du non-être, puisque dans le Sophiste l'enjeu est justement celui d'une définition «phénoménologique » du statut de la vérité du langage, en référant ce dernier à la définition des deux types d'images et des deux genres d'imitation. 
L'appropriation, le saisissement de l'objet par le logos n'est qu'une modalité de marquer la transitivité du langage et sa distance, en même temps qu'une modalité de désigner le véritable « objet » que l'on s'approprie dans l'exercice de cet acte, à savoir l'identité de celui qui proferre la connaissance de toute chose donnée.

Formulée brièvement, une première réponse de Platon au sujet de cette question centrale de l'identité de l'être en tant qu'homme, serait alors la suivante : est homme, digne de ce nom, celui qui, en s'adressant à un autre, est capable de tout dire de ce qu'il pense à l'égard d'un être, d'un objet ou d'une action, mais sans s'identifier lui-même à ce «tout » comme si, de fait, il rapportait toujours à l'autre les paroles d'un tiers; comme si aucune parole ne lui appartenait en entier alors que c'est bien lui qui la profère comme expression de sa pensée. Est homme celui qui sait donc qu'il n'est pas tout ce qu'il sait, tout ce dont il a le savoir par la vision, par la pensée et par l'usage de l'expression; celui pour qui l'identité avec soi est encore ailleurs que dans les idées et les paroles (noemata kai logoi) dont il a la mâ̂trise, mais à l'égard desquelles il a acquis aussi le savoir de s'en extraire et de se tenir à l'écart pour une raison précise qui reste à définir. Cette raison, on la devine néanmoins : elle provient de la limite atteinte de l'expression, de l'impossibilité de tout dire de ce que l'homme ressent et connait, de ce savoir négatif socratique - je sais que je ne sais pas; je désire m’instruire parce que je n'ai pas la connaissance de ce qui doit être connu, à commencer par moimême. Mais la conséquence de cette situation projette son ombre bien plus loin : l'homme peut avoir et peut manifester ainsi l'intuition que la part d'inconnu qui motive et nourrit son élan cognitif représente peut-être la part la plus importante qui soit, dès lors qu'elle ménage la place pour ce qui demeure irréductible dans l'expérience directe, et irréductible aussi à toute expression immédiate ou différée. L'accomplissement de la connaissance est donc atteint lorsque la limite de la connaissance est elle-même atteinte et assumée comme immanente à la fois à l'objet et au sujet. Cette «méthode » de Platon inspirera durablement la démarche des Humanistes, à proprement parler, bien que ces derniers aient tenté aussi de l'harmoniser avec les positions d'Aristote, de la Bible, des Pères de l'Église et des théologiens médiévaux. « Se connaître, en effet, c'est tout connaître en soi (qui enim se cognoscit, in se omnia cognoscit) - dit Pic de la Mirandole dans Oratio De hominis dignitate -, comme l'ont écrit d'abord Zoroastre, puis Platon dans Alcibiade. », car « $\gamma \nu \tilde{\omega} \theta_{l} \sigma \varepsilon \alpha v \tau \dot{\omega} v$, idest cognosce te ipsum, ad totius naturae nos cognitionem... excitat et inhortatur - 'connais-toi toimême' nous incite et nous exhorte à l'étude de la nature entière ${ }^{10}$. » 
Mais cette réponse de Platon à la question de l'homme à travers les possibilités conjointes de l'opsis, du noein et du legein maîtrisées avec art et appliquées à la recherche de l'identité de chaque chose en soi, réponse dont l'humanisme s'est fait l'écho retentissant, n'est pas complète. Platon va plus loin dans la définition. Il précise à cet égard un point de vue différent, voire nouveau par rapport à celui visé par Socrate dans la pratique dialectique, et par rapport à l'objet abstrait qui est au centre de l'ontologie métaphysique de Parménide. L'affirmation de l'être prend en effet place dans l'homme et se dit pleinement de celui qui sait aimer et sait démontrer, de surcroît, que c'est bien cela que d'être homme : aimer en dépassant les limites conjoncturelles d'une connaissance réduite à la possession de l'objet, et sans en référer pour cela aux principes ; savoir, en outre, en parler puisque parler est un art qui n'est pas seulement transitif mais aussi conatif, servant à la maîtrise des passions, et, en somme, un art dont la pratique certifie pour l'homme la possibilité de tenir l'équilibre exact entre les forces opposées qui déterminent par attraction et rejet l'existence physique du monde pour les Anciens. Parler aux bêtes, parler aux dieux - voici en deux mots extrêmes comment la « ruse » du logos, celle qu'Ulysse maîtrisait déjà, se transforme dorénavant en vertu qui définit pour Platon l'homme qui aime au-delà de l'objet, l'homme qui, dès lors qu'il aime ainsi l'amour, connaît aussi ses forces et se connaît non comme un objet mais comme un être capable de se déterminer par ses limites. Or, il me semble que l'audace d'une telle position philosophique ne sera relevée véritablement qu'à la Renaissance, quand la découverte textuelle des Dialogues croisera des idées analogues nourries par la tradition médiévale des poètes. La réaction se fera attendre, mais ne sera pas moins provocatrice dans les temps modernes que n'a été puissant le défi de cette audace relevé par les Humanistes, audace qui consiste à considérer l'homme comme l'unique vivant capable d'affirmer son amour sans nécessité d'en faire la preuve par un acte violent.

Levons un premier voile de cette approche théorique en prenant une série d'exemples textuels. Elle concerne l'objet spécifique de l'amour pour Platon $^{11}$. Aucun mystère n'entoure d'ailleurs cet objet dès lors que j'ai prononcé au départ le nom de l'un des deux dialogues consacrés principalement à définir et à louer l'amour par l'entremise des mots, des phrases et de l'intonation des discours. L'objet de l'amour dont il est question dans le Phèdre comme dans le Banquet, bien que différemment présenté, se décline

11. Précisons que notre article n'est pas consacré à la définition des deux faces de l'amour platonicien, eros et philia, thème vaste et qui dépasse nos intérêts dans ce contexte, mais au discours spécifique qui prend comme thème l'amour et cherche le langage approprié à sa définition. 
en trois temps : l'amour de tous les corps qui ont la jeunesse naturelle pour leur beauté, puis l'amour d'un seul corps, beau entre tous parce qu'il permet de voir ce qu'il y a de plus beau que la beauté d'un corps, à savoir la beauté de l'âme $\left(\kappa \dot{\alpha} \lambda \lambda \lambda_{0} \tau \tau \mu \iota \dot{\omega} \tau \varepsilon \rho \circ \nu\right)^{12}$, pour arriver à aimer la beauté tout court, la beauté sans support étranger, sans corps, car identifiée à elle-même éternel-

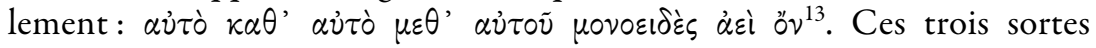
d'amours sont les degrés d'initiation au « mystère » de l'amour unique, tels que Diotime les avait un jour présentés à Socrate, lequel les rapporte dans le Banquet en citant le propos de la prêtresse de Mantinée, les paroles d'une étrangère, comme une «initiation » à l'amour à travers l'éloge composé dans les règles de l'art ${ }^{14}$. Ces trois degrés de l'amour correspondent aux trois types de connaissance que Platon expose ailleurs sous des modalités différentes, mais dont le modèle de la République est bien sûr le plus connu et sera déterminant pour la postérité du Maître: la connaissance intuitive, fournie par une «bonne vue » (elle-même sujette à un certain apprentissage), la connaissance discursive issue d'une parfaite maitrise de l'art du logos (une techne qui s'apprend moyennant quelques efforts auprès d'un instructeur patenté), et enfin la connaissance contemplative, laquelle permet de saisir l'identité de l'objet en soi en ouvrant ainsi la possibilité de se saisir soimême dans cette expérience réflexive requise par l'accès à l'identité de l'objet de la connaissance. Cette dernière connaissance - à savoir : la possibilité d'un accès à l'identité de l'être en soi, unique, de chaque chose ainsi



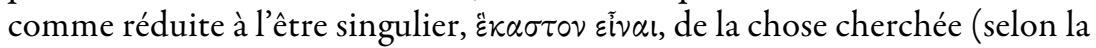
leçon clairement exposée dans le Théétète, 153e-154a) - comprend une particularité par rapport aux deux autres: elle ne s'apprend pas comme telle, n'est pas semblable à un «art » acquis auprès d'un maître qui exerce un métier spécifique à l'objet de l'enseignement. Elle prend acte et se parfait d'elle-même dans le sujet lui-même, à condition que celui-ci ait choisi la bonne voie dans la vie en ayant été conduit par un autre vers ce chemin : $\tau \grave{o}$

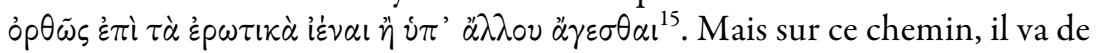
soi, chacun se retrouve seul.

Une injonction, voire une exhortation est faite à toutes les étapes au néophyte entré dans le « mystère » de l'institution amoureuse ( $\tau \tilde{\omega} \nu$ हे $p \omega \tau-$ -

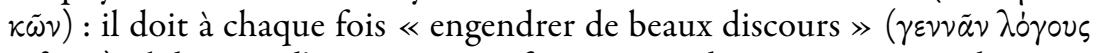
$\kappa \alpha \lambda \circ \dot{s} \varsigma)$. Il doit, en d'autres termes, faire preuve de sa puissance en donnant naissance à cet « être animé », à cet être de substitution en quelque sorte

12. Symp. $210 \mathrm{~b}$.

13. Ibid. $211 \mathrm{~b}$.

14. Ibid. $210 \mathrm{a}-211 \mathrm{~d}$.

15. Ibid. 211c. 
par rapport à un être animé véritable - en enfantant donc, à chaque fois qu'il franchit une étape, un beau discours. Or le discours tient ici la place du rejeton vivant, de l'enfant dans lequel le néophyte devrait pouvoir se retrouver, mais qu'il ne lui est pas donné d'engendrer puisqu'il s'agit sur ce parcours d'une union dont l'aimant est à l'évidence le seul partenaire, comme un sujet laissé en suspens, l'acte prévalant ici sur l'objet de la même manière que le langage recouvre l'expérience directe de la chose réelle mais ne se l'approprie pas.

Les «beaux discours » permettent ainsi de découvrir d'abord la

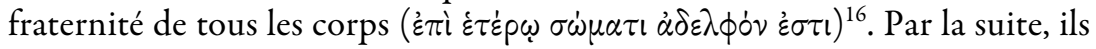
permettent de distinguer cette fraternité purement formelle de la parenté véritable, celle qui est au-delà du sang ${ }^{17}$ tout autant qu'au-delà des formes ressemblantes, et qui unit tout en ramenant l'individu à l'identité avec lui-

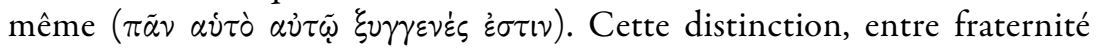
formelle et parenté essentielle, devient possible après que l'on ait encore

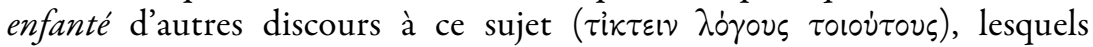
louent, par exemple, la belle âme cachée dans un corps dont la présence est

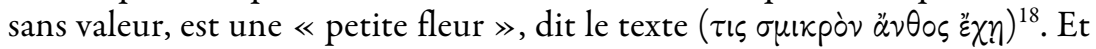
encore d'autres multiples, beaux et grandioses discours devront ainsi être

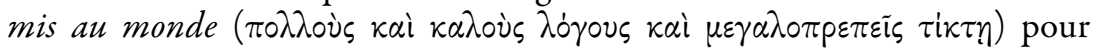
exprimer les pensées qui proviennent de l'élan insatiable vers la sagesse,

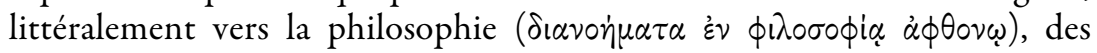
discours portant sur l'universalité du beau, discours engendrés lorsque le

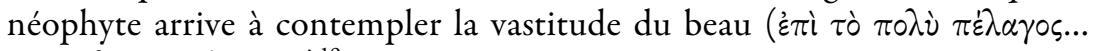

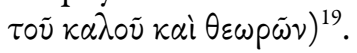

On comprend quel est finalement l'objet de l'amour pour Platon : c'est le Beau, bien sûr, la beauté universelle saisie par la contemplation de tout, beauté qui engendre en celui qui la contemple l'élan vers le Bien, élan confondu, pour les humains, avec l'élan vers la philosophie - élan ou excitation dont parle Pic de la Mirandole dans le passage cité plus haut ; «fureurs socratiques que chante Platon dans le Phèdre (Socraticis illis furoribus, a Platone in Phaedro decantatis) $\gg$, dit encore le même jeune platonicien de la Renaissance dans un autre passage de son Oratio $^{20}$. Mais cet élan n'aboutit pas à un pouvoir de possession de l'objet par le savoir, car il est comme détourné du chemin droit vers l'objet visé. Il peut en revanche, grâce à cet

16. Ibid. $210 \mathrm{~b}$.

17. Platon lui-même définit ainsi l'amitié comme une parenté au-delà du lien naturel, le lien du sang, dans la Lettre VII, $324 \mathrm{~d}$ et $350 \mathrm{~b}$.

18. Symp. 210b-c.

19. Ibid. $210 \mathrm{~d}$.

20. Oratio De hominis dignitate, trad. Hersant 1993, p. 33. 
infléchissement, accomplir sa véritable mission. Pourquoi ce détour de la voie directe est-il nécessaire ? Parce que seul un dieu peut déceler directement l'unité dans tout, selon un passage dont le Timée (68d) offre une formule brève et claire ${ }^{21}$, alors qu'un homme, même instruit et éclairé par l'initiation, n'a aucun pouvoir comparable de distinction ni, en outre, aucune possibilité de posséder ce qui lui est donné ainsi à contempler dans l'indistinction écrasante du multiple à laquelle il a accès à ce stade de la connaissance. Il aime et connaît jusqu'à un certain point son objet, mais, en fin de compte, sans pouvoir y toucher. Il peut cependant, et doit même d'ailleurs, enfanter des discours, mettre en acte des paroles et les maîtriser par l'art de la composition, comme on engendre, on aime et on instruit ses enfants, même si, ici, il s'agit littéralement d'enfanter des paroles et de les mettre en acte dans un discours construit. Autrement dit, le discours sert ici de substitut à l'objet de la connaissance, dont la possession entière est réservée au dieu seul. Mais, par le même acte, l'homme s'avère en possession de ce que le dieu n'a pas, à savoir la maîtrise du logos et le pouvoir d'exprimer et d'exposer ainsi le meilleur de sa nature, son âme et son intelligence des choses, les deux «incarnées » dans une expression qui le représente parfaitement et ne s'identifie toutefois pas à son être entier.

Un détour est donc signifié de cette manière : il est nécessaire d'accompagner l'initiation à l'objet véritable de l'amour par un acte vrai de création, car ce dernier à la fois décentre l'homme de lui-même et lui permet en même temps de se retrouver et de s'accomplir de cette manière, en ayant bouclé la boucle de toutes ses possibilités. L'amour en sortira grandi puisque l'homme aura atteint la complétude de pouvoir qui constitue le propre de son identité : c'est ainsi qu'il sera entièrement lui-même dès lors qu'il a lui-même engendré, c'est-à-dire qu'il a pu se mettre dans les conditions de l'acte dont l'objet est celui même qu'il désire et qu'il cherche, et dont il réalise, comme sujet, une re-création sur un autre support, celui de la parole et du discours. Même s'il ne s'agit que de créer des similitudes (l'art de la parole est comme toutes les technai soumis au règne de la vraisemblance), engendrer des beaux discours doit néanmoins participer comme acte d'une création vraie et entière. La complétude signifie alors le dépassement de la limite inhérente à la nature. La création d'un objet propre à l'homme, et à lui seul, se substitue ainsi à l'objet inatteignable du désir par ce détour. Le rôle du discours est de faire justement le jeu de cette substitution, mais sans en être pour autant lui-même l'objet du désir. Dans le processus de la connaissance platonicienne - appelons ce processus élan vers la philosophie

21. « Car seul un dieu sait bien comme on peut mêler en un même tout, pour les dis-

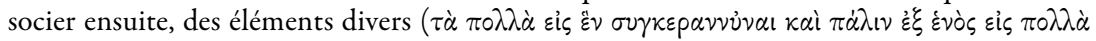
$\delta(\alpha \lambda \dot{\varepsilon \varepsilon v}$ ), et seul il est aussi capable de le faire. » (Tim.68d, trad. Rivaud 1925.) 
et vers la contemplation du Beau qui assure la connaissance du Bien - le discours opère donc le passage depuis l'objet vers l'acte : polariser l'acte d'aimer à défaut d'une possession impossible de l'objet. Toute possession véritable étant impossible, autant que la connaissance propre du singulier s'avère impossible au sein du multiple, ou que la possession de l'identité en soi d'une forme universelle est considérée comme impossible à l'individu humain, l'acte de mise en paroles de l'amour constitue dans ce contexte un modèle pour la manière dont cette impossibilité peut être contournée et dont la connaissance s'accomplit sans en être amoindrie ou obligée de procéder par réduction. Dire l'amour exprime l'acte même d'une création accomplie sur le coup de l'expression. Pour cette raison, dire l'amour devient exemplaire pour tout acte conjoint de la parole qui montre et qui signifie la connaissance. Car un art accompli du langage désigne en effet plus que les paroles ne disent proprement, dès lors que le discours parfait vise non une maîtrise de l'objet par la connaissance (sur le modèle archaique du pouvoir de la nomination), mais la possibilité d'une maîtrise de son identité par la maîtrise du sens juste des mots qui montrent cet objet et le signifient en même temps. En somme, par la maîtrise de l'acte de signifier, par la dynamis propre au logos manifestée dans le feu de l'action, dans l'exercice de la parole comme une image volontaire, et parfaite autant que possible, de l'acte d'aimer.

De même que le Bien, impossible à atteindre, se reflète dans le Beau et peut ainsi être saisi, la connaissance de tout objet, impossible comme identité, se reflète dans la signification que le langage découvre et que l'homme peut saisir en « fabriquant », amoureusement, des discours parfaits. Mais retenons que pour Platon la condition de l'amour est centrale pour le langage, comme la contemplation (ou pensée spéculative) est pour lui la condition centrale pour la vraie connaissance. Pourquoi l'amour serait-il la condition centrale (et première en quelque sorte) du langage ? Parce que le langage est foncièrement un acte qui n'agit pas directement; c'est un acte de la médiation perpétuelle, qui a par conséquent besoin de substituer à la pure transition d'une vérité de paroles un milieu actif à partir de soi, une puissance génératrice capable de donner aux mots une force d'impact que seuls le souffle des mots et l'écho de la musique parfaite des sons ne peuvent pas offrir au meilleur des discours qui soit. Art de signifier, le langage n'est pourtant pas un code de signes (d'où la méfiance de Platon à l'égard de l'écriture), dès lors que chaque mot est une limite assumée par la pensée à l'égard de l'expérience dans laquelle s'exerce l'humain. Que le langage puisse être codifié par la suite jusqu'à la dernière de ses figures, cela est une autre histoire qui mérite l'attention, mais dont il ne sera question ici que tangentiellement, dans le rapport entre usage dialectique et usage rhétorique de la parole. 
La composition de discours représente ainsi la condition propre à l'homme ; une forme de retrait, certes, mais aussi sa seule condition de possibilité par rapport à l'avancée d'une connaissance qui n'est donnée selon Platon qu'aux dieux, et dont l'homme a acquis à ce stade un savoir aussi précieux que décevant. Il sait désormais que la connaissance ne lui est pas donnée par une possession complète et immédiate d'aucun objet, mais par l'unique voie du reflet, et qu'il se doit de maîtriser ce reflet, et de le maitriser réellement, faute de quoi il risque de perdre tout savoir, et même de mettre, tel Narcisse, sa vie en péril. Mais une fois arrivé là, l'homme, qui a appris qu'il ne possède vraiment rien, se connaît lui-même comme réfléchi à la fois par cette altérité transcendante à l'égard de son identité et par son acte de parole qui le représente pleinement à l'égard d'autrui. Il découvre ainsi l'identité du « sujet » (qu'il nous soit permis, et pardonné, de recourir à cet inévitable anachronisme terminologique). Cette découverte s'opère lorsque l'homme capable de parler assume et s'approprie ses paroles comme un langage de l'amour et comme un moyen de se découvrir dans chaque être ou objet comme dans une altérité butoir pour soi-même. S'il aime toutefois passionnément ce qu'il connaît de cette manière, et s'il donne naissance, engendre l'objet propre de son acte d'aimer, la beauté recherchée lui apparait alors réfléchie sans faille dans un discours parfait : un éloge ou un poème - pour Socrate. Que ce discours soit une définition de l'objet visé en même temps qu'une louange, ce n'est pas anodin mais il s'agit, encore une fois, d'une question de genre que je ne traiterai pas ici, bien qu'elle soit déterminante pour une certaine finalité assignée par Platon au langage. Aussi a-t-il été aisé de reconnaître la synthèse que je viens d'opérer entre le discours de Diotime rapporté par Socrate dans le Banquet, la leçon à Alcibiade (autour de l'injonction de Delphes, dans l'Alcibiade) et la succession des trois discours dans le Phèdre; une allusion aussi au sort du prisonnier initié de retour dans la caverne, dans la République VII, prisonnier éclairé qui s'exerce dans l'art de la rhétorique pour échapper au courroux de ses compagnons et pour leur enseigner, malgré eux, la distinction des genres entre les ombres et le principe des réalités. Dans chacune des circonstances citées, c'est la voix de Socrate qui s'entend dans le texte de Platon.

Si j’ai évoqué les propos de Diotime relayés par Socrate dans le Banquet, propos sur lesquels je reviendrai, c'est pour souligner ce singulier assemblage platonicien entre théorie et pratique du langage qui suppose que la connaissance soit nécessairement rythmée et conditionnée par l'exercice des discours, par la mise en acte réitérée de l'art du logos. De la conjonction entre la recherche du beau et le témoignage (par l'acte de la parole, donc) que ce désir de beau est bien présent dans le choix du discours approprié, naissent à la fois les beaux discours et l'amour parfait, sans que l'on sache exactement qui détermine qui ou quoi. Quoiqu'il en soit, ces discours, 
reflets du désir d'aimer, doivent être, comme les « vivants » (hosper zoion), issus d'une rencontre amoureuse. Les discours sont cependant étranges comme «vivants », puisque ce sont des « vivants » qui n'ont pas besoin d'un support extérieur à leur être dès lors qu'ils sont le fruit d'une rencontre d'incorporels: la rencontre entre l'acte de bien penser et l'acte de bien parler - tous deux issus de l'élan d'un acte unique, celui d'aimer, acte qui constitue une expression ou une forme d'ostension de l'âme. Aucune inversion, ni aucun désaccord n'est toléré dans cette relation construite sur la réciprocité du reflet. Mais le but visé n'est pas d'arriver à engendrer le discours parfait, l'expression la plus adéquate à une pensée qui maîtrise superbement l'objet dont elle a acquis par contemplation le savoir. À moins, certes, d'en faire son métier. Le but de cette « institution amoureuse », à laquelle Diotime initie Socrate, est de faire naître dans l'initié un homme nouveau, un être vivant digne de ce nom ; de faire naître, en effet, l'homme accompli dans l'être initié.

L'union entre l'acte de penser et l'acte de parler ( alors que la première des conditions. Il faut avoir rempli avec désir et grâce le devoir de cette union de la pensée et du langage, c'est-à-dire en aimant ce devoir comme Socrate qui déclare à Phèdre s'être entiché des divisions et

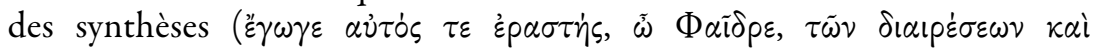

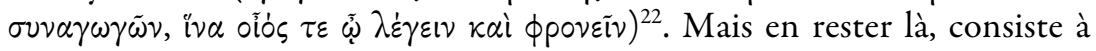
s'arrêter sur le seuil d'un acte qui ne produit alors que du vent. Devenu capable de saisir cette aptitude dans l'autre - aptitude à viser l'unité et à la saisir lorsqu'elle se trouve naturellement déployée dans une multiplicité -, il importe maintenant d'arriver à l'imiter. Non à la manière des peintres, mais plutôt à la manière des poètes. Il faut, en effet, pouvoir se mettre avec son esprit sur les traces de cet autre et à son pas, en le suivant comme s'il

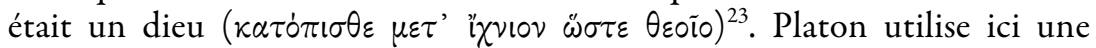
expression rythmée empruntée à Homère - Ulysse suit les pas d'Athéna ou de Calypso - ou du moins, Platon fait entendre des mots à la manière

\section{Phaedr. 266b.}

23. Ibid. 266b : «je marche sur ses pas, je le suis à la trace comme un dieu ». Socrate cite ici, de manière quasi littérale, une occurrence relativement fréquente chez Homère : Ulysse en train de suivre les pas, donc l'agissement et la volonté d'Athéna et non pas la sienne propre; une seule fois il suit aussi Calypso, de la même manière que sa déesse tutélaire. Le passage est important dans l'économie du Phèdre, car Socrate y définit la méthode dialectique comme moyen de donner la possibilité de parler et de penser ( $\lambda \dot{\varepsilon} \gamma \varepsilon \iota \nu \tau \varepsilon$ kai $\phi \rho o v \varepsilon i v)$ par le biais de deux opérations du langage : la division et l'unification ou rassemblement, opérations qui permettent d'articuler par les vertus du logos l'un et le multiple. Voir pour les occurrences dans l'Odyssée les références citées par L. Brisson (dans Brisson 1997, n. 358, p. 224) et, pour un commentaire de ce passage concernant la mise en place de la méthode dialectique, Dixsaut 2001, p. 105.) 
d'Homère pour signifier que Socrate, ayant parlé ainsi d'amour à l'égard de la dialectique, ne peut désormais qu'emprunter aussi le nom d'un poète qui agit différemment à l'égard de la langue et va sans doute plus loin que lui. Le poète, en effet, divise et unit à son tour les contraires, mais arrive à le faire autrement que par des arguments de mots, des hypothèses et des démonstrations, puisque sa fin est différente de la propédeutique des savoirs dévolue aux philosophes. La parole homérique est théologique ; pour accomplir son but elle chante, raconte et donne à voir. Le passage évoqué du Phèdre (266b) se trouve, on le devine aisément, à la jointure entre les genres, en l'occurrence à la charnière entre les deux arts du langage : la définition de la dialectique et celle de la rhétorique.

Mais les deux arts du langage ont pour Platon un but commun. Lorsque dans le Phèdre Socrate décrit donc le bon discours, celui qui est semblable à un vivant, il l'anthropomorphise au même moment où il le profère; il le présente ainsi presque comme un double de l'orateur qui le compose et le récite $^{24}$. Si le discours est construit avec quelque « ruse (mechane ou metis) à la place de l'art accompli du genre, s'il peut se lire à l'envers par exemple, tel l'épitaphe de Midas le Phrygien ${ }^{25}$, ou s'il peut se reprendre au beau milieu des phrases pour se contredire, ou peut se réciter plusieurs fois pour défendre des buts différents, l'âme du logos «vivant » est alors tordue, monstrueuse, incontrôlable par la raison, dépassant le champ de la vision tout autant que le domaine strict du logos, le domaine de la signification. Le langage ne révèle plus l'objet, ni ne confronte la connaissance avec les limites propres de l'expression. Il résonne, certes, mais les sons seuls qui s'entendent, même articulés et compréhensibles lexicalement, ne suffisent pas pour signifier la vie, car ils ne montrent ni ne transmettent rien qui vaille dans ce cas. Poussés dans ce retranchement du vivant, les mots régressent en babils - des sons qui n'ont pas de référent même si l'on reconnaît le nom d'une chose ou les stridulations d'un chœur de cigales -, ou alors demandent que l'on assigne un contenu discursif à des expressions comme « chant des Muses » ou « voix du daimon », sachant toutefois que celles-ci ne réfèrent à rien d'existant dans l'immédiat. En revanche, être capable d'《enfanter des discours », plus exactement pouvoir donner naissance à un discours qui ait un corps, une tête, des pieds, et que toutes ces parties soient assemblées entre elles de manière à ce que le tout soit con-

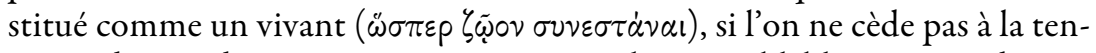
tation de prendre tout ceci pour une métaphore semblable aux précédentes,

24. Pour une exégèse de ce thème précis du discours comme « vivant », en l'occurrence l'assimilation du logos à un zo(i)on dans le Phèdre, mais aussi avec des références au Timée, au Banquet et à la République, voir l'article de Brisson 1987.

25. Phaedr. $264 \mathrm{~d}$. 
cette capacité constitue alors l'indicateur d'un processus réflexif dont l'objet n'est pas le logos comme tel mais bien l'homme qui parle. Celui-ci met en acte la parole et engendre ainsi sa propre identité, celle qui a la possibilité d'être transmise ou montrée sans danger de produire un dédoublement trompeur comme un portrait ressemblant mais sans vie. Tout acte de parole réfère à son agent et le met sur une scène commune avec l'objet visé. Capable d'enfanter son identité, c'est-à-dire de la manifester en définissant l'objet de son « dit », et d'être ainsi lui-même auprès des autres, l'homme devient en quelque sorte un homme « nouveau », un homme accompli dans sa nature. C'est bien lui que cherchent à obtenir Diotime par initiation à l'amour et Socrate par accouchement de l'esprit ou par initiation à la dialectique. Si l'on ne cède pas le terrain à la métaphore - et je ne vois ici aucune raison philologique ou philosophique de le faire puisque hoion ou hosper sont des indicateurs de comparaison sur fond de ressemblance formelle ou structurelle et non nécessairement de transfert sur un autre support -, le discours « anthropomorphe » que loue Platon dans le Phédre, devient alors, tout simplement, le portrait vivant de l'orateur accompli.

Revenons encore au Banquet. Vers la fin du discours de Diotime rapporté par Socrate, la contemplation du beau en lui-même, selon luimême et par lui-même dans une identité unique et éternelle avec soi (aj̉ò

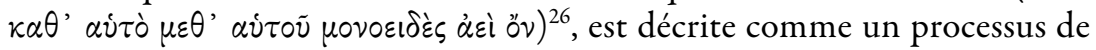
désengagement à la fois à l'égard des distinctions et à l'égard des identifications formelles. À l'inverse de la manière dont est décrit le discours vivant dans le Phèdre, le candidat à la perfection dans les choses amoureuses

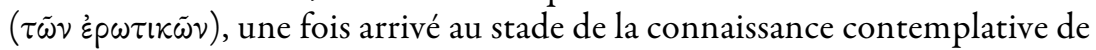
l'océan du beau, doit se défaire non seulement de toutes les choses belles ( $\tau \grave{\alpha}$ $\kappa \alpha \lambda \dot{\alpha})$ qu'il s'est précédemment appropriées en les contemplant, mais aussi des habitudes prises et qui l'ont conduit jusqu'à ce stade, à savoir : la possibilité de distinguer l'accroissement et le décroissement, les parties belles des parties qui ne le sont pas, ainsi que les jugements divergents des uns et des autres sur l'aspect des choses - en un mot, il doit reléguer l'art des distinctions, la dialectique, à son but et ne pas la généraliser en s'arrêtant là. Puis, il doit également se défaire de l'analyse qui procède par représentations $(\phi \alpha \nu \tau \alpha \sigma \theta \dot{\eta} \sigma \varepsilon \tau \alpha$ l) et éliminer tout ce qui pouvait ressembler pour lui à un visage (oĩov $\pi$ pó $\sigma \omega \pi \circ \nu$ ), à des mains ou à toute autre partie d'un corps

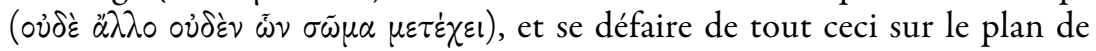
l'ontologie, de la réalité de ces choses existantes en quelque altérité que ce

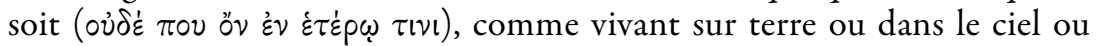

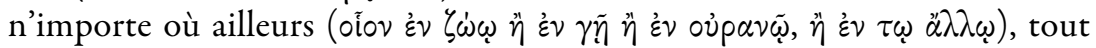


autant qu'il doit se défaire des représentations sur le plan du discours et du

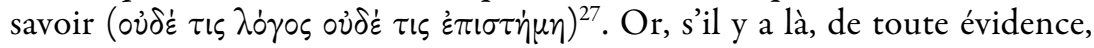
un renversement exact de la manière dont le discours doit être composé par abstraction et non à la ressemblance d'un vivant comme dans le Phèdre -, c'est parce que l'objet de l'amour auquel se réfère Socrate dans le Banquet est différent même si le but de l'acte linguistique reste, à mes yeux, le même.

L'art d'aimer et l'art de produire des discours appropriés visent tous les deux leur achèvement devant l' $\mathrm{l}^{\prime}$ «océan du beau ». Ils vont du même pas et suivent le même chemin. Seulement, le premier s'applique à l'être et le signifie en tant que celui-ci est par rapport à un référent extérieur, et qu'il ne constitue donc jamais la cause ni le principe de lui-même, tandis que le second vise la pensée comme telle, en acte grâce au langage, or le langage, bien appris et bien utilisé, constitue l'expression juste et droite de la raison dont il procède. Le second est en outre téléologique tandis que le premier ne l'est pas. À ce titre, le langage semble pouvoir se suffire à lui-même, voire semble pouvoir se détacher, s'autonomiser en quelque sorte comme signification par rapport au sujet, telle une âme, si celle-ci pouvait se dire, pouvait exprimer elle-même son identité, et se montrer ainsi séparée du corps. Le langage agit ainsi comme s'il était animé, se mouvant de lui-même. Mais, tant que l'acte de la pensée s'identifie à l'acte de dire (legein) en même temps qu'à la signification des mots (rhemata) et du logos, le sujet qui parle a besoin d'un intermédiaire pour se saisir soi-même comme son propre objet, ou alors doit accepter de se mettre sur les traces d'un autre que luimême, emprunter la trace de ses pas et son rythme, à défaut de se substituer à l'autre en lui empruntant son corps. Diotime, encore elle, demande à Socrate de faire de son mieux l'expérience de ses paroles à elle auprès de son

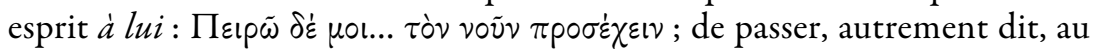
crible de son intellect les mots qui pourraient venir d'ailleurs, pourraient être « inspirés » chez elle. Après tout, cette femme, prêtresse et étrangère, pourrait-elle avoir raison, seule et contre tous ? Il faut des preuves - ellemême, d'ailleurs, les demande à son ami. Il faut exercer ou pratiquer en esprit la puissance révélatrice d'un discours; y voir aussi les conséquences jusqu'au bout.

Autrement dit, savoir exprimer sa pensée en des paroles parfaites consiste à savoir déterminer son véritable objet et à parler de lui en s'adressant à autre chose qu'à l'aspect que l'on peut avoir d'emblée sous les yeux : le visage d'un interlocuteur, aussi aimable soit-il, ou la face des choses, aussi rayonnante soit-elle que la lumière de l'été. Mais il faut aussi savoir maîtriser le pouvoir d'autonomie que les mots acquièrent ainsi, les tenir toujours 
auprès de soi en connaissant leurs effets pour les avoir vécus ou assumés soimême en tant qu'«actes » véritables avant de les adresser comme «parole » à autrui. La réflexivité antique joue justement sur ce levier archaïque entre l'acte de dire et l'acte de faire en le plaçant - chez Platon encore comme chez les poètes des mythes cosmogoniques - sur le terrain d'une pensée de la puissance, de la détermination totale et de l'accomplissement par l'eros. Pour cela, il fallait bien que le discours soit comme un vivant, qu'il soit engendré avec un corps, une âme et un lien qui assure l'harmonie de l'ensemble. Mais il fallait assurer aussi la possibilité de faire passer par ce « corps de paroles » la pensée déterminante, et donc donner une structure à la connaissance en accord avec le but recherché, comme si on pouvait avoir une sorte de connaissance préalable de celui-ci, le vérifier par des preuves ou par l'expérience : en termes anciens, aimer par principe avant de connaître la détermination exacte de l'identité par rapport à tel être singulier, savoir, autrement dit, ce qu'il est dès lors qu'il est là, sous les yeux, dans les parages. L'aimer aussi pour pouvoir ou savoir en parler, du moins s'assurer la condition pour cela.

Aussi pourrait-on formuler cette position philosophique en des termes anachroniques certes pour Platon, mais qui peuvent nous éclairer, nous, dans la perspective de l'histoire de la pensée, sur l'enjeu de cette position platonicienne à l'égard du statut du langage comme enjeu spécifique et comme gage d'identité de l'être humain. L'instrument universel vise ainsi, et toujours, les transcendantaux, mais la parole, qui s'entend, se voit et s'adresse à autrui en le touchant, dévoile dans son élan quelque chose à l'égard du concept en soi : il s'agit de la signification, du sens qui ramène le concept vers l'être qui parle hic et nunc et oblige ce dernier à travailler avec les noms et les concepts à partir d'un horizon métalinguistique, en cassant ainsi le mécanisme d'autoréférence propre au langage (conséquent à sa transitivité) et en ouvrant la parole à l'acte en présence, et, il va de soi, à la maîtrise de la puissance persuasive engendrée par le discours. La conséquence immédiate en est l'amorce d'un changement radical de la théorie de la vérité du langage telle qu'elle était entendue et pratiquée par les sophistes du temps de Platon. Le statut du langage quitte alors l'alternative ontologique être-paraître, désignée jusque-là comme une opposition essentielle pour déterminer l'accès à la vérité par le logos, et se positionne dorénavant dans la complémentarité entre immanent et transcendant - une complémentarité à l'égard de laquelle le langage n'occupe plus toute la surface de la connaissance possible des choses qui existent ou qui n'existent pas, mais laisse désormais une place à ce qui serait la possibilité d'une altérité radicale, silencieuse et ineffable. Voir par exemple, dans le Sophiste, le passage qui 
conclut la démonstration concernant le rapport entre langage et image ${ }^{28}$, ainsi que le processus de désontologisation et de désubjectivation du discours (sophistique, en l'occurrence), processus illustré par la demande explicite de désimplication pronominale des interlocuteurs dans la recherche d'une définition du langage propre au sophiste ${ }^{29}$. Nous avons dans ce cas affaire à une situation qui représente l'inverse exact de ce qui aurait un sens, signifierait quelque chose, mais ne montrerait rien - ce que Barbara Cassin appelle la « possibilité-limite d'une autonomie signifiante » dans le cas d'une théorie aristotélicienne de la signification (sous l'exemple classique du bouc-cerf) $)^{30}$.

Revenons encore aux textes de Platon. L'art de la parole en tout adéquate à la pensée doit par conséquent passer entièrement, se résorber presque dans l'art d'aimer pour qu'une recherche de la connaissance de l'identité en soi de l'être puisse mener l'homme jusqu'au rivage de la Beauté. Lorsque Socrate, se déclarant amoureux d'Alcibiade, tente de le définir pour pouvoir maîtriser cet être beau entre tous par une connaissance parfaite de ce qu'il est, il place son ami dans un lieu propice à saisir les ressemblances, le lieu, catoptrique par excellence, où l'être se reflète luimême dans et par son propre désir. Pour Socrate ce lieu n'est autre que le langage exprimant en des paroles bien choisies le désir amoureux capable d'engendrer des discours. Mais Socrate ne s'adresse précisément pas à la présence physique d'Alcibiade ni même à son visage. Pour souligner la nature véritable de la relation qu'il entend établir avec lui, il ne tente pas de le décrire. Il joue au contraire sur la négation de l'apparent, tout autant que sur la sonorité des mots, comme s'il regardait l'objet de son amour à dessein de biais, ou en se détournant, tel le démiurge du Timée (28a-b) cherchant à saisir le modèle de la création cosmique ailleurs qu'au bout de ses doigts ${ }^{31}$.

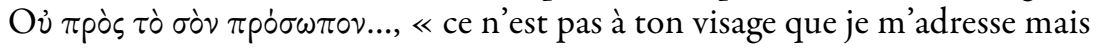

28. Soph. 241a.

29. Ibid. $238 \mathrm{~d}-239 \mathrm{~b}$.

30. « Parle si tu es un homme », dans Cassin \& Narcy 1989, p. 40.

31. «Toutes les fois donc que l'ouvrier, les yeux sans cesse fixés sur ce qui est identique [ayant le regard tourné éternellement vers l'identique; ou encore, ayant pour toujours établi

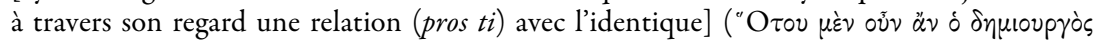

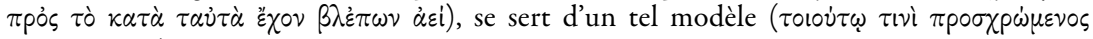
$\pi \alpha p \alpha \delta \varepsilon i \gamma \mu \alpha \tau \iota)$, toutes les fois qu'il s'efforce d'en réaliser dans son œuvre la forme et les

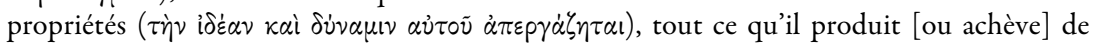

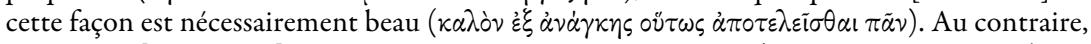

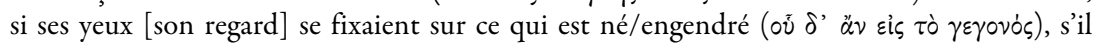

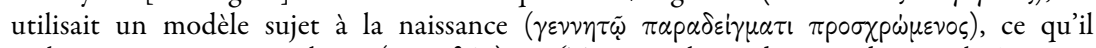

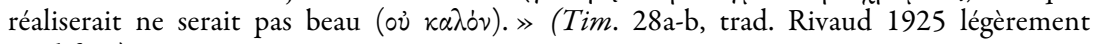
modifiée.) 
à toi-même; c'est à ton âme que je parle et non à ce que j'ai devant les yeux », dit Socrate à Alcibiade, non sans un évident jeu phonétique $\pi$ pòs $\tau$ ò бòv $\pi \rho \dot{\sigma} \sigma \omega \pi \circ v$, tel un effet d'écho, semblable à celui qui se produit inévitablement dans la relation de face-à-face, par l'envisagement direct - $\pi$ pòs

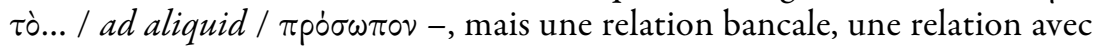
ce qui demeure muet pour l'instant, comme mort. En écho, donc, parce qu'à ce stade Socrate dit et Alcibiade acquiesce et répète aveuglément presque les mêmes mots. Il s'agit de la préparation à la connaissance de soi par le regard plongé dans le regard d'un autre (Alcibiade, 130e). Mais Socrate insiste lourdement sur la nécessité d'établir l'identité de l'autre, comme pour le réveiller à lui-même en suscitant le désir : il faut distinguer avant

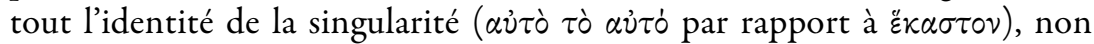
pour rejeter l'une au profit de l'autre, mais pour établir l'adresse juste de la parole dans la recherche de la connaissance - ici : savoir qui est véritablement celui qu'aime Socrate, lui le premier.

De ce que nous disions tout à l'heure : qu'il fallait chercher d'abord ce que

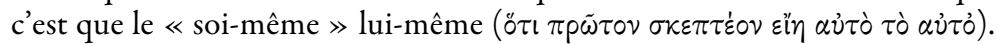
Or au lieu du « soi-même » considéré absolument, nous avons cherché ce

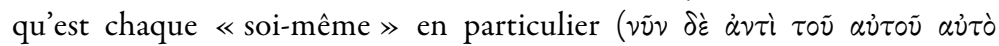

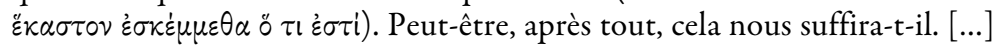
En conséquence... quand nous nous entretenons, toi et moi, en échangeant

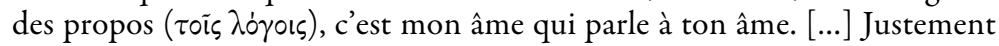
comme nous le disions à l'instant: quand Socrate s'entretient avec Alcibiade par un échange de propos, ce n'est pas à ton visage qu'il parle, mais

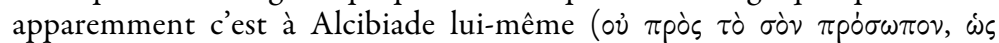

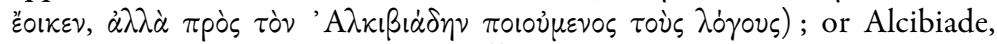
c'est ton âme ( $\tau 0 \tilde{v} \tau \circ \delta \dot{\delta} \dot{\varepsilon} \sigma \tau \iota \nu \dot{\eta} \psi \nu \chi \dot{\eta}){ }^{32}$

Inutile de préciser qu'en parlant ainsi d'Alcibiade, Socrate s'adresse quand même à Alcibiade, même s'il feint de s'en détourner ${ }^{33}$. Et le jeu continue sur le même ton, ironique certes, et sur la même structure de la composition des arguments, en énumérant tout ce qui pourrait constituer un trompe-l'œil de l'identité si l'art de la parole, bien aiguisée par la précision de la pensée, ne savait pas détacher le sujet de l'objet afin d'éviter toute confusion et toute emprise : le nom, le corps et les parties du corps, le

32. Alc. 130 d-e (trad. Croiset \& Desclos 1996).

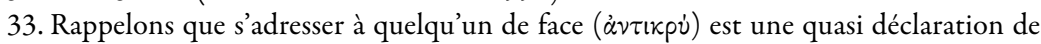
guerre, ou en tout cas l'expression d'un affrontement de contraires dans le monde grec classique. On n'envisage directement un être, homme ou dieu, que dans des situations particulières, bien établies. Rappelons aussi, par ailleurs, qu'Alcibiade fut pendant toute l'histoire de l'Académie classique le dialogue qui ouvrait le cycle de l'enseignement des doctrines platoniciennes, en l'occurrence l'enseignement de la théorie gnoséologique. 
métier exercé, la fortune propre etc. À l'œuvre, la connaissance discursive précède nécessairement l'acte d'aimer.

Je voudrais enfin lever, pour finir, un second voile dans cette approche, toujours à propos de la définition que j'ai formulée au début : est homme celui qui sait aimer et sait dire que c'est bien cela que d'être homme et d'être dans le monde. Platon donne la meilleure preuve à cet égard dans le Phédre en composant trois discours dans les deux genres (dialectique et rhétorique), sur le thème de l'amour.

Il ne s'agit pas, pour Socrate qui parle et agit, de concilier les genres du logos; ni de substituer logos à eros comme une forme de fougue à une autre forme de fougue, en laissant croire à Phèdre que la maîtrise de soi s'obtient par un art enseigné à l'école assorti du devoir accompli en récitant ou en se rappelant religieusement des mythes. Platon met en œuvre et décrit la manière dont est engendrée et se définit au cours d'un discours l'identité de celui qui parle par rapport à celui qui écoute. Il décrit comment se définit cette identité de l'être en situation d'acte de parole, même lorsque l'orateur la cache, volontairement ou à son insu.

Après avoir écouté Phèdre le brillant lire, le visage empourpré de plaisir, le discours matinal de Lysias, et après lui avoir répondu par une réfutation composée à son tour dans les règles de l'art dialectique, Socrate se dévoile et parle à nouveau pour se purifier : $\kappa \alpha \theta \dot{\eta} p \alpha \sigma \theta \alpha \iota \dot{\alpha} \nu \dot{\alpha} \gamma \kappa \eta$ - dit-il cette fois-ci ${ }^{34}$. Un péché contre la mythologie vient d'être commis ou frôlé dans les deux discours prononcés : celui de Lysias, rapporté par Phèdre, et celui de Socrate en réponse au premier, mais ne parlant pas en son nom propre. puisqu'il choisit de se couvrir la tête comme s'il s'absentait. Qu'est-ce qu'un péché en matière de mythologie ? Et comment doit se purifier quiconque aurait commis une telle faute ? Il y a dans ces cas, dit Socrate, une purifica-

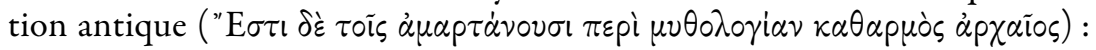
retourner le mal en bien par le même moyen, i.e. celui de la mythologie par les vers, le récit, les images. La faute entraîne la cécité, tandis que le repentir ouvre à nouveau les yeux. C'est l'histoire de Stésichore qui le montre. Je ne la répéterai pas ici car elle est bien connue ${ }^{35}$. O

\section{Phaedr. 243a.}

35. Stésichore retrouve la vue après avoir nié le récit de la trahison d'Hélène ; Homère, qui n'a pas connu ce remède antique, est resté aveugle toute sa vie. Non, ce n'est pas Hélène qui a trahi la couche de Ménélas, c'est son fantôme (phantasma), une représentation, car le langage, à ce propos, n'a aucun pouvoir, n'a littéralement aucune signification. Pour une interprétation récente des passages d'Homère, de Platon et d'Euripide concernant la nature du double d'Hélène, fantôme, figure d'air (eidolon d'aither) ou « statue de nuage » ( $\nu \varepsilon \phi \dot{\varepsilon} \lambda \eta \varsigma$ $\ddot{\alpha} \gamma \alpha \lambda \mu \alpha$ ), en rapport avec le statut du langage et la logique symbolique de l'œuvre - voir Bettini 2004 (en particulier p. 228-230). 
$\lambda$ óyos oũtos - dire ne signifie pas d'emblée quelque chose, ne signifie même rien (au sens étymologique des mots), n'a pas de sens lorsqu'on cherche à prêter ainsi à des dieux l'outrance qu'à aucun prix un homme n'accepterait à son propre égard : manifester le contraire de son essence. Les mots tiennent leur sens premier de l'acte référé ou signifié par le sujet ${ }^{36}$. Pécher contre la mythologie, c'est faire violence au langage sous couvert d'histoires invraisemblables, comme si le monde (celui des humains comme celui des dieux) était seulement un fait de paroles (wording the world... ${ }^{37}$ ) pour le meilleur ou pour le pire, et que l'on pouvait lire l'un à travers l'autre en appuyant une démonstration sur des arguments herméneutiques tirés du cru de sa propre fiction. Pécher contre la mythologie, c'est en faire mauvais usage, et en outre, confondre les genres : prendre le récit pour une analogie, par exemple, et ajuster la narration à une relation de proportions qui ne convient qu'à la connaissance structurelle des choses et non à des êtres qui se manifestent par des actes. Les mots, bien qu'ils soient parfois les mêmes, ne recouvrent pas un sens identique quand ils affirment un état immanent des choses ou quand ils racontent une histoire de dieux et de héros. Malin serait alors celui qui trouverait des raisons humaines et des désirs dans l'agissement des dieux pour en faire autant, sans dévoiler l'illusion de ce langage puisqu'il est dupe ou mal intentionné (allusion au Phèdre $262 \mathrm{a}^{38}$ ). C'est une mauvaise question que de savoir si des histoires comme celle d'Orithye et de Borée sont vraies ou fausses, tel ce mythologème ( $\tau \grave{o} \mu \nu \theta_{0-}$ $\lambda \dot{o} \eta \eta \mu \alpha)$ qui avait intrigué Phèdre, en arrivant sur le bord de l'Ilissos au sanctuaire d'Agra, et que Socrate, agacé par le bavardage de son compagnon, avait jeté par-dessus bord en envoyant les agrestes figures tératologiques au pâturage des esprits entichés de cette sagesse simplette qui cherche la vérité dans les interprétations des figures et des noms ( $\dot{\alpha} \mu \eta \chi \dot{\alpha} \nu \omega \nu$

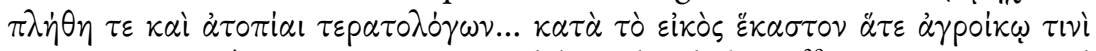

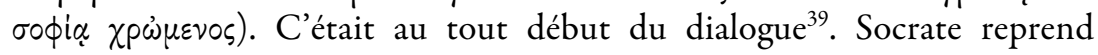

36. Précisons que parler ici à son propre compte ne veut pas dire parler de soi sous le mode épique (i.e. raconter ses propres exploits). Au sujet de Stésichore justement, et du langage «littéraire » antique qui met en scène un rapport non narratif mais lyrique de l'auteur à soi, voir Schneider 1993 (en particulier p. 24).

37. Allusion à $S$. Cavell ; voir l'épigraphe de cet article.

38. «Il faut donc, alors, si l'on doit faire illusion à autrui ( $\dot{\alpha} \pi \alpha \tau \dot{\eta} \sigma \varepsilon เ \nu \mu \dot{\varepsilon} \nu \ddot{\alpha} \lambda \lambda \circ \nu)$, mais sans être soi-même dupe de l'illusion ( $\alpha \dot{u} \tau \dot{\partial} v \delta \dot{\varepsilon} \mu \grave{\eta} \dot{\alpha} \pi \alpha \tau \dot{\eta} \sigma \varepsilon \sigma \theta \alpha \mathrm{l}$ ), que l'on connaisse à fond

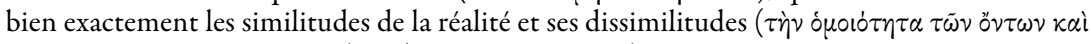

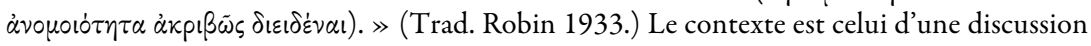
concernant les discours dans les tribunaux et l'éloquence politique.

39. Phaedr. 229 c-e. C'est cette même question agaçante : est-il ou serait-il vrai que... ? qui vaut le refus de l'interprétation par Socrate au début du Phèdre, et que dénonce Austin 
maintenant son esprit et, purifié du «péché contre la mythologie », tient alors le troisième discours du texte de Platon, un discours tissé presque entièrement de mythes et faisant au départ l'éloge du délire, avec cette frappante «fureur socratique » qui a fasciné tant d'esprits depuis la Renaissance (en particulier Pic de la Mirandole parmi les premiers).

Mais Socrate ne parle toujours pas en son nom : il rapporte le logos de Stésichore ${ }^{40}$. Plus précisément, il commente le premier vers que j'ai cité et ouvre ainsi les yeux de son compagnon sur la valeur sémantique et référentielle du langage épique, en s'étant assuré auparavant que Phèdre était bien là, qu'il était attentif et présent en écoutant Socrate de son mieux. Comme dans Alcibiade ${ }^{41}$, Socrate s'adresse à Phèdre en parlant de Phèdre, comme s'il pouvait y avoir un décrochement entre l'identité de son interlocuteur immédiat et une image ou un mauvais rêve, un eidolon ou un phantasma, décrochement dû au moins à quelque maladie des yeux (maladie qui d'ailleurs sera évoquée plus loin ${ }^{42}$ ), si ce n'est à un défaut de l'âme.

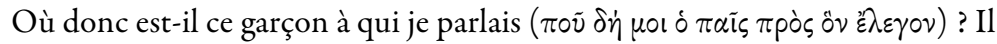

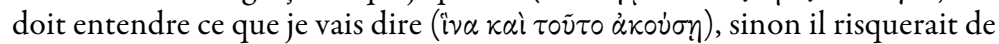
prendre les devants et de donner ses faveurs à qui ne l'aime pas. Il est près de

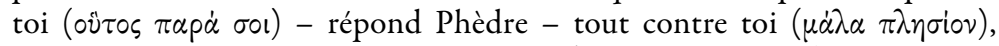

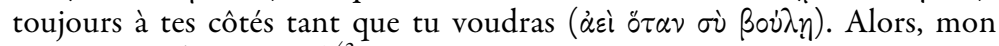
beau garçon $(\tilde{\omega} \pi \alpha \tau \kappa \alpha \lambda \dot{\varepsilon})^{43} \ldots$

Phèdre tient désormais le langage de l'aimé et Socrate peut l'aimer et parler de l'amour en présence. (Honni soit qui mal y voit ou pense.)

Voici maintenant comment doit s'exprimer son discours [le discours de Lysias, redressé a posteriori par Socrate] : «il n’y a pas de vérité dans un

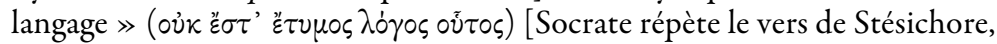
que je préfère traduire, les raisons en sont évidentes ! : il n'y a pas de sens

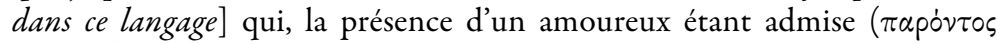

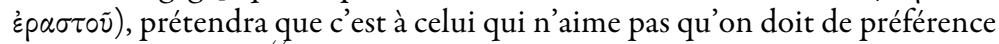
accorder ses faveurs ${ }^{44}$...

Pour aimer, comme pour enseigner et apprendre, l'acte concerne l'être en présence et le langage ne peut pas et ne doit pas dans ce cas jouer le rôle d'un substitut ontologique comme lors de la connaissance d'un objet en soi. Socrate s'en explique sur le long et étonnant parcours qui suit.

dans l'analyse du langage ordinaire («Plaidoyer pour les excuses », Austin 1994, p. 148 et n. 6).

40. Phaedr. 244a.

41. Passage cité supra.

42. Phaedr. 255d.

43. Ibid. 243e (traduction Vicaire 1998).

44. Ibid. $244 \mathrm{a}$. 
Après avoir parlé de manière détournée du langage et de l'homme, sous l'espèce du délire et de l'immortalité de l'âme, en louant le langage du premier et en brossant l'image bien connue du cocher et des deux chevaux pour illustrer les agissements de l'âme, faute de pouvoir définir cette dernière autrement que par l'acte du mouvement par soi, principe du mouvement éternel, Socrate en arrive à la question initiale de son discours : il doit définir l'amour. Il doit pour cela entrer dans le jeu, se mettre lui-même en jeu, pour définir l'identité de l'acte et de l'objet, après avoir composé, pour se purifier, son propre mythe (lui-même appelle mythos le récit du cocher qui conduit l'attelage et dompte le cheval révolté). Or Socrate passe alors d'un régime de langage à un autre et nécessairement d'un registre visuel à un autre registre de visibilité. Il annonce d'ailleurs ceci par la référence (métaphorique ?) au danger d'aveuglement, ou d'une possible maladie des yeux. Le «mythe de l'âme » est censé avoir défini et montrer l'acte, le verbe même d'aimer (eran), દ̇pã., dit le texte, il aime maintenant (indicatif présent de la troisième personne). Le discours doit désormais faire autant à

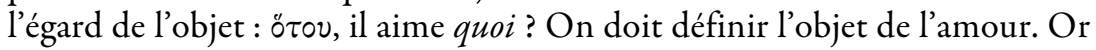
la réponse, et d'ailleurs la solution envisagée par Socrate, consiste à mettre en place, comme dans l'Alcibiade, le mécanisme catoptrique de l'amour dans l'acte même de la parole proférée. Le passage est célèbre parce qu'il est cité et commenté aussi bien par Plotin (Ennéade I, VI, 8) que par les sophistes tardifs, comme Philostrate par exemple (dans Eikones, le « tableau $\gg$ de Narcisse).

Comme un souffle (oĩov $\pi \nu \varepsilon \tilde{\nu} \mu \alpha)$ ou un son ( $) \times \dot{\omega}$ - l'écho, à proprement parler) renvoyé par une surface lisse $(\lambda \varepsilon i \omega \nu)$ et solide $(\sigma \tau \varepsilon p \varepsilon \tilde{\omega} \nu)$ revient à son point de départ, le flot de la beauté retourne vers le bel enfant en passant

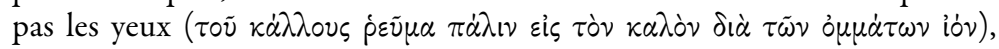
chemin naturel de l'âme; il atteint celle-ci, la remplit, arrose le passage des ailes et les fait pousser, et remplit à son tour d'amour l'âme du bien-aimé. Il aime donc, mais il ne sait quoi. [...] Comme un homme qui a pris une ophtalmie à un autre, il ne peut en dire la cause, et il oublie qu'il se voit luimême, dans son amoureux, comme dans un miroir ( $\omega \sigma \pi \varepsilon \rho \rho \dot{\delta} \dot{\varepsilon} \nu \kappa \alpha \tau \dot{\partial} \pi \tau \rho \omega$

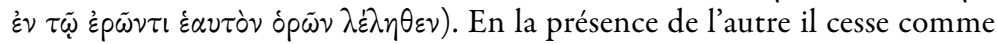
celui-ci de souffrir, en son absence il éprouve les mêmes regrets, et il est regretté de la même façon : il éprouve un 'contre-amour', image réfléchie de

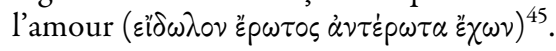

Ainsi l'identité de l'être engendré par le discours devient-elle, sur le parcours, l'identité d'un être aimé réfléchi dans et par la présence de son amant, comme l'être constitue pour la pensée un tout, inséparable de l'altérité du non-être lorsqu'il s'agit de distinguer, pour les définir, la réalité 
des choses elles-mêmes de la vérité du langage qui est comme une « contreréalité », en miroir, à leur égard. Car le langage a beau être pareil au phantasma d'Hélène pour Stésichore ${ }^{46}$, a beau constituer donc un hosper zo(i)on, un simili-vivant seulement, il représente néanmoins l'unique moyen pour l'homme de donner aux choses véritables leur sens, et par là une consistance ontologique et non seulement une épaisseur sémantique. Le détour qui s'engage devient dès lors un témoignage d'amour par l'engendrement des discours, c'est-à-dire par la capacité de composer un éloge à même de porter l'élan de l'homme vers ce qu'il n'est pas, vers cette non-identité qu'il désire connaître et qui peut, en réponse, engendrer la connaissance de soi tout autant que de l'altérité visée par l'amour. L'acte de connaître est bel et bien dans ce cas un acte d'amour : à ce titre il transcende la réalité immanente et ne peut, à ce titre, qu'être divin - mais divin à hauteur d'homme, car c'est l'homme lui-même, accompli par et dans ce processus, qui devient divin, qui devient «autre » puisqu'il n'est pleinement lui-même qu'au terme d'un acte d'initiation de l'ordre d'une traversée de ses propres limites.

Le passage conclusif du Phèdre (278b-d), juste avant la prière adressée au dieu Pan, est particulièrement éclairant sur le rôle du langage et sur la nécessité d'un usage approprié à l'objet pour pouvoir définir celui qui parle, et surtout pour pouvoir donner un sens à ses paroles, faire en sorte que le langage signifie en référant à quelque chose de vrai : acte, être ou objet.

Assez joué maintenant à propos des discours ( $\tau \dot{\alpha} \pi \varepsilon p i \lambda \partial \dot{\gamma} \omega \nu)$. Toi, va trouver Lysias. Dis-lui qu'étant descendus ( $\kappa \alpha \tau \alpha \beta \dot{\alpha} v \tau \varepsilon)$ tous deux jusqu'au ruis-

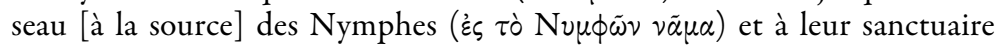

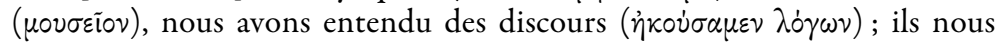
invitaient à dire à Lysias et à quiconque compose des discours, puis à Homère et à quiconque compose de la poésie, aussi bien sans mélodie que chantée, enfin à Solon et à ceux qui, dans l'ordre des discours politiques ont rédigé des écrits sous le nom de lois: «Si l'un de vous a composé ces

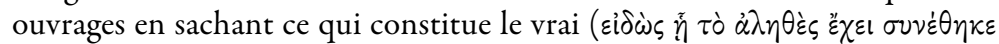
$\tau \alpha \tilde{\tau} \tau)$, s'il peut leur porter assistance en affrontant la discussion [la réfutation] sur ce qu'il a écrit ( est capable, par la parole, de montrer lui-même que ses écrits sont peu de

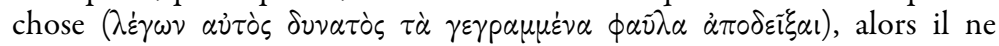
faudra point nommer un homme de cette qualité d'après ses œuvres d'écrivain, mais d'après l'objet supérieur [la visée] qu'il a poursuivi [avec sérieux]

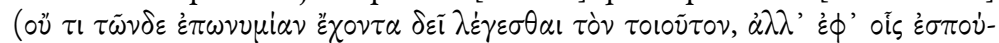

46. Le thème est repris dans l'Hélène d'Euripide, v. 540 sq. L'épisode de la rencontre entre Ménélas et Hélène donne l'occasion d'un jeu, presque platonicien, sur les ressemblances et les dissemblances. C'est dans ce contexte qu'apparaît cette formule célèbre : @è̀s

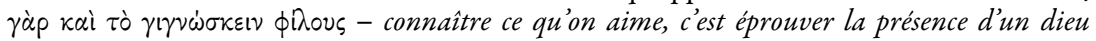
(v. 560). Pour le rapprochement entre amour et amitié, voir encore le Phédre, 255e. 


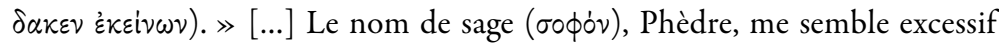

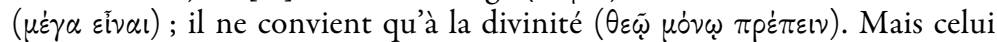

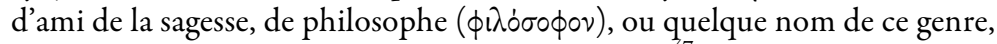
lui conviendrait mieux, et serait d'un ton plus juste ${ }^{47}$.

Ce passage, célèbre entre tous, précise dans un premier temps la nature du trajet effectué par les deux philosophes : c'est une katabase vers la source des Nymphes, en l'occurrence vers la source qui anime et alimente sans cesse l'inspiration, comme la physis anime et nourrit la vie. Mais plus précisément, puisqu'il s'agit de la mise en œuvre des possibilités du logos, cette source désigne le «moteur » de l'inspiration, l'élan, le désir suscité d'emblée par la beauté naturelle, physique. Socrate et Phèdre vont donc là où se dévoile le désir (le lieu naturel «absolu » sous la figure de la jeune fille Orithye $^{48}$ ) et où sort de sous terre, au grand jour, ce qui est naturellement beau et qui nourrit la possibilité de mettre ce désir en acte (par l'amour) et en ouvre (par le chant ou par le discours), les deux, amour et chant, ou discours, en même temps, comme si l'acte du langage et l'acte qui traduit le désir (ou la volonté) pouvaient et devaient même être simultanés dans leur détermination réciproque. Sont ensuite récapitulés les genres de l'art du langage : discours sophistique (Lysias), discours politique (Solon), poème et récit (Homère). Puis Socrate met l'accent sur l'enjeu central de toute production de langage: savoir définir le vrai ( $\tau \dot{\alpha} \alpha \lambda \eta \theta \theta \dot{\varepsilon} \varsigma$ ), savoir ce qui est « vrai » et le viser lors de toute composition afin de rendre ainsi en tout lieu la chose présente en vérité. Osons gloser : viser le vrai constitue la raison de toute production de synthèse par le moyen de deux opérations complémentaires, la réduction du multiple et l'unification par le langage, puisque ce dernier a d'une part la possibilité de distinguer les oppositions et de l'autre de viser la notion commune à tout (ou notion universelle) sous chaque identité déterminée.

Vient ensuite le tour d'une définition nécessaire de la méthode, laquelle consiste à mettre le langage en situation d'affronter ses limites: par la négation (ou réfutation) des thèses et par l'acceptation de la limite elle-

47. Trad. Vicaire légèrement modifiée.

48. Remarquons aussi que le lieu choisi par Socrate pour écouter le discours de Lysias et mener le dialogue avec Phèdre est situé non seulement dans la proximité de la source des Nymphes et du lieu où la belle Orithye fut enlevée dans la fougue de Borée, le Vent méchant qui enlève les âmes, mais aussi sous un platane et dans le voisinage d'un gattilier en fleur. Ce dernier symbolise la maîtrise de la fécondité, la stérilité et la chasteté ; selon la tradition populaire les feuilles de gattilier permettent de contenir le désir érotique et sont utilisées dans les pratiques liées aux cultes des dieux dans les villes d'Attique. Le dialogue sur la rencontre entre la rhétorique et la dialectique au sujet de l'amour et du beau est ainsi d'emblée mis sous le signe d'une rencontre initiatique, d'une sorte de descente du langage à la source des possibilités de la vie et de la mort. 


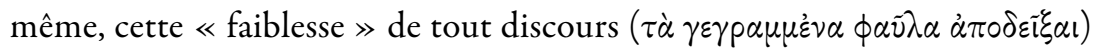
à l'égard de l'identité, du visible et du mouvement. Cette méthode, ou plutôt cet «art » par lequel la pensée devient langage, est évidemment la dialectique. Son but constitue le dépassement de l'œuvre (et donc de la définition de l'homme comme artisan producteur d'une œuvre) par la mise en lumière du véritable enjeu : l'objet qui est vraiment visé, avec sérieux -

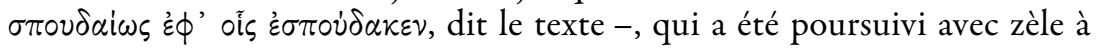
travers l'œuvre, cet objet-là ne s'identifie pas à l'œuvre ni ne se trouve dans celle-ci mais ailleurs. Où ? La réponse de Socrate est ici indirecte. Si le but visé coïncide avec l'œuvre, cet « objet » du dépassement de l'acte est alors nécessairement circonscrit à la perfection du chant, du discours sophistique ou de la rédaction des lois ${ }^{49}$. Dans ce cas l'ouvrier du logos n'est pas différent des autres ouvriers doués et diligents, qui accomplissent avec art leurs métiers, qui produisent des objets ou des œuvres et qui prennent par conséquent le nom de leur métier en s'y identifiant : prince-gouverneur ou cordonnier. Mais si le but est supérieur à l'œuvre, le maître de la parole vraie tend alors vers une coïncidence avec le divin par la sagesse, ou, toute proportion gardée, dit Socrate malicieux, avec ce qui au moins se situe dans la puissance de l'amour du divin et de la sagesse, en l'occurrence, la philosophie. C'est ailleurs que Platon (toujours sous couvert de Socrate) donne une réponse directe à cette question clef. Le passage 176b-c du Théétète reprend de près le propos final de Socrate dans le Phèdre mais situe cette fois-ci le rôle du philosophe et du langage philosophique dans le rapport entre le bien et le mal : la connaissance de leur différence est « sagesse

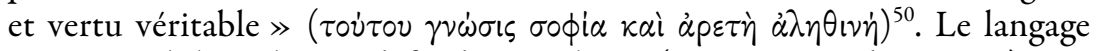
vraiment philosophique, défendu par Platon (sous couvert de Socrate), vise ainsi à mettre les «transcendantaux » (le Beau et le Bien) au cœur même et non à l'horizon de l'immanent, dans l'agir propre au domaine de l'éthique et non à la lisière d'un arrière-monde des Idées impossible à atteindre. Mais même sans la précision ajoutée par le Théétète, le passage conclusif du Phèdre que nous venons de citer pourrait constituer le condensé d'un véritable «art philosophique »: le «manifeste » platonicien des enjeux que représente la mise du langage à hauteur d'homme.

On ne peut pas accuser Platon, comme on le fait couramment, d'avoir obligé la pensée philosophique à détourner son regard de l'être singulier, puisque c'est bien celui-ci, l'être singulier, qui aime et qui est capable d'en parler, en faisant la preuve de son amour non par un geste de possession mais par un acte de parole adéquat à la vérité du sens donné à l’amour.

49. Il s'agit du passage $278 \mathrm{~d}$-e, qui fait suite immédiate à celui que nous venons de citer. 50. Théétète, $176 \mathrm{c}$ (trad. Diès 1926). 
L'être singulier se détermine alors dans l'œuvre non seulement de manière grammaticale, comme sujet désigné par le nom propre ou par le pronom, mais aussi, et surtout, en faisant la preuve que ce qu'il dit est bel et bien ce qu'il pense et ce qui meut son choix et son agir. L'amour joue son rôle à la fois nourricier et moteur précisément là, dans l'audace de cette transparence de bon aloi entre la pensée et l'expression, dans la transparence d'un pouvoir décentré de lui-même, qui entraîne l'être à viser par la parole une vérité en acte. Mais c'est le langage qui tient le rôle d'un plan catoptrique pour l'être et qui signale aussi ce point d'infléchissement radical (appelé conversion, epistrephein) permettant à celui qui parle de se saisir luimême et de se mettre sur le droit chemin. La connaissance de soi qui s'ensuit, et qui s'appellera «conscience » quelques siècles plus tard chez les disciples gréco-romains de Platon ${ }^{51}$, est en point d'orgue ce qui tient attachées dans les Dialogues l'épistémologie et l'éthique, à savoir la maîtrise des passions, en un mot: la vertu. C'est elle, et plus exactement la transformation de la connaissance en vertu, la métamorphose du «savoir » en « faire le bien » qui permet de connaître son identité non comme un étant existentiel confondu dans le multiple, tel qu'il est, existentiellement parlant, pour le maître de l'Académie, mais comme l'être propre et singulier à qui il appartient d'agir en accord avec la pensée et la parole proférée, quitte à mettre sa vie en péril comme le prisonnier libéré, de retour dans la caverne.

On n'est pas obligé, me semble-t-il, de considérer l'œuvre platonicienne sous un angle strictement anthropologique, extérieur à la méthode dialectique, pour comprendre ce dessein philosophique qui est aussi, à vrai dire,

51. On considère Apulée comme le premier à associer le terme conscientia au démon intérieur (De Deo Socratis, XVI, 156). Voici le texte: «Ainsi donc, vous tous qui écoutez par mon truchement cette divine théorie de Platon, sachez bien, en vous disposant à chacune de vos actions et de vos réflexions, qu'avec de tels gardiens l'homme ne peut avoir aucun secret ni dans son cour ni au dehors: le démon s'immisce dans tout avec curiosité, inspecte tout, se rend compte de tout, descend au plus profond de nous, comme la conscience. Ce gardien privé dont je parle, gouverneur personnel, garde du corps familier, curateur particulier, enquêteur intime, observateur inlassable, spectateur inséparable, témoin inévitable, improbateur du mal, approbateur du bien... » Proinde uos omnes, qui hanc Platonis diuinam sententiam me interprete auscultatis, ita animos uestros ad quaecumque agenda uel meditanda formate, ut sciatis nibil homini prae istis custodibus nec intra animum ne foris esse secreti, quin omnia curiose ille participet; omnia uisitet, omnia intellegat, in ipsis penitissimis mentibus uice conscientiae deuersetur. Hic, quem dico, priuus custos, singularis praefectus, domesticus speculator, proprius curator, intimus cognitor, adsiduus obseruator, indiuiduus arbiter, inseparabilis testis, malorum inprobator, bonorum probator... (Beaujeu 1973, nous soulignons). Le contexte néo-pythagoricien dans lequel est formé Apulée à Athènes, vers le milieu du $\mathrm{II}^{\mathrm{c}}$ siècle, explique le décentrage du précepte socratique et le rôle accordé dans ce contexte au daimon. 
un dessein à visée politique ${ }^{52}$. On n'est pas obligé non plus d'appeler ce dessein du nom, certes forcé, d'humanisme avant la lettre. Mais il me semble qu'il y a dans les textes suffisamment d'arguments et de preuves philologiques et philosophiques qui vont dans ce sens, à condition d'aimer nous-mêmes la lettre et son usage pour y voir le sens. À condition aussi d'accepter la possibilité d'un dépoussiérage massif de l'œuvre platonicienne, en la sortant de sous le carcan unique de la théorie des Formes immuables et de la séparation du sensible et de l'intelligible dans laquelle l'ont enfermée les siècles d'un enseignement de la doxa, ainsi que l'histoire longue d'un jugement de la philosophie sous le signe d'une alternative entre l'acceptation sans condition ou le refus en bloc d'un «platonisme scolaire $»^{53}$ transformé en doctrine.

La mise en acte de la parole est avant tout une manière d'assumer l'expérience métalinguistique de l'identité de soi et d'autrui comme une limite qui s'exprime quand dire signifie affirmer, statuer et instancier la présence de l'être qui est concerné sans en faire pour autant son objet - j'entends: l'objet d'une possession de l'être et du monde qui le manifeste. Il me semble que Platon est le premier philosophe qui en a eu conscience et qui l'a exprimé, même s'il n'a pas bâti là-dessus tout son « système ».

52. Voir dans ce sens les remarques de L. Brisson sur l'enjeu de la Lettre VII comme une sorte d'«Apologie de Platon », de première tentative d'autobiographie dans l'histoire du genre, et de testament politique en même temps : Brisson 1993.

53. L'expression «platonisme scolaire » appartient à J. Pépin qui l'utilise de nombreuses fois (Pépin 1964). 


\section{BIBLIOGRAPHIE}

Austin, J.L. 1994 : Écrits philosophiques, Paris, 1994 (La couleur des idées). [Traduction, par L. Aubert \& A.-L. Hacker, de Philosophical papers, Oxford, 1961.]

Baslez, M.-Fr., Ph. HoffMAnN \& L. Pernot (éd.) 1993 : L'invention de l'autobiographie : d'Hésiode à Saint Augustin. Actes du deuxième colloque de l'Équipe de recherche sur l'hellénisme post-classique (Paris, École normale supérieure, 14-16 juin 1990), Paris, 1993 (Études de littérature ancienne, 5).

Beaujeu, J. 1973 : Apulée, Opuscules philosophiques (Du dieu de Socrate; Platon et sa doctrine; Du monde) et fragments, texte établi, traduit et commenté par-, Paris, 1973 (Collection des Universités de France).

Bettini, M. 2004 : «Construire l'invisible: un dossier sur le double dans la culture classique $\gg$, Métis, N.S. 2 (2004), p. 217-230.

BRISSON, L. 1987 : « Le discours comme univers et l'univers comme discours: Platon et ses interprètes néo-platoniciens », dans: Le texte et ses représentations, Paris, 1987, p. 121-128 (Études de littérature ancienne, 3).

- 1993: «La Lettre VII de Platon, une autobiographie?», dans: M.-Fr. Baslez, Ph. Hoffmann \& L. Pernot 1993, p. 37-46.

- 1997 : Platon, Phèdre, traduction inédite, introduction et notes. Suivi de: Jacques Derrida, La Pharmacie de Platon, édition augmentée d'un supplément bibliographique (1992-1996), Paris, 1997 (GF, 488).

Cassin, B. \& M. NARCY 1989: La décision du sens: le livre Gamma de la Métaphysique d'Aristote, introduction, texte, traduction et commentaire, Paris, 1989 (Histoire des doctrines de l'Antiquité classique, 13).

Chiron, P. 2002 : Pseudo-Aristote, Rhétorique à Alexandre, texte établi et traduit par -, Paris, 2002 (Collection des Universités de France).

Croiset, M. 1920 : Platon, CEuvres complètes, I : Introduction. Hippias mineur; Alcibiade; Apologie de Socrate; Euthyphron; Criton, texte établi et traduit par -, Paris, 1920 (Collection des Universités de France).

Croiset, M. \& M.-L. Desclos 1996 : Platon, Alcibiade, traduction par M. Croiset, introduction et notes de M.-L. Desclos, Paris, 1996 (Classiques en poche, 4).

DIÈs, A. 1926 : Platon, CEuvres complètes,VIII.2 : Théétète, texte établi et traduit par -, Paris, 1926 (Collection des Universités de France).

Dixsaut, M. 2001 : Métamorphoses de la dialectique dans les dialogues de Platon, Paris, 2001 (Bibliothèque d'histoire de la philosophie).

Hersant, Y. 1993 : Giovanni Pico della Mirandola, De la dignité de l'homme = De hominis dignitate, traduit du latin et présenté par -, Combas, 1993 (Collection Philosophie imaginaire, 20).

VICAIRE, P. 1985 : Platon, CEuvres complètes, IV.3 : Phèdre, texte établi par C. Moreschini et traduit par P. Vicaire. Notice de Léon Robin, Paris, 1985 (Collection des Universités de France).

PÉPIN, J. 1964 : Théologie cosmique et théologie chrétienne (Ambroise, Exam. I, 1, 1-4), Paris, 1964 (Bibliothèque de philosophie contemporaine).

Rivaud, A. 1925 : Platon, CEuvres complètes, X: Timée; Critias, texte établi et traduit par -, Paris, 1925 (Collection des Universités de France).

Robin, L. 1933 : Platon, CEuvres complètes, IV.3 : Phèdre, texte établi et traduit par -, Paris, 1933 (Collection des Universités de France).

SCHNEIDER, J. 1993 : «Usage de la première personne et autobiographie dans la poésie lyrique archaïque », dans : M.-Fr. Baslez, Ph. Hoffmann \& L. Pernot 1993, p. 2136. 